\title{
Curves and surfaces of spacelike curves according to Bishop frame and their singularities
}

\author{
Haiming Liu ${ }^{\mathrm{a}}$, Jiajing Miao ${ }^{\mathrm{a}}$, Donghe Peib,* \\ a School of Mathematics, Mudanjiang Normal University, 157011 Mudanjiang, P. R. China. \\ ${ }^{b}$ School of Mathematics and Statistics, Northeast Normal University, 130024 Changchun, P. R. China.
}

Communicated by D. Baleanu

\begin{abstract}
Legendrian dualities between pseudo-spherical images of spacelike curves in Minkowski 3-space are investigated by using the theory of Legendrian duality. Moreover, the singularities of parallel lightcone developables, dual surface, Bishop pseudospherical Darboux images and Bishop pseudo-spherical images, which are generated by spacelike curves, are classified from the viewpoints of wave fronts and caustics, and we also give some more detail descriptions on the conditions of those singularities. Finally, some properties of parallel slant helix are given. (C)2017 All rights reserved.
\end{abstract}

Keywords: Spacelike curves, Minkowski 3-space, Bishop frame, Legendrian dualities.

2010 MSC: 32S25, 53A35.

\section{Introduction}

Bishop [2] introduced that there exists an orthonormal relatively parallel adapted frame, which we call Bishop frame, other than the Frenet frame and compared features of them with the Frenet frame. The Bishop frame has many properties that make it ideal for mathematical research and computer graphics [8-10, 20]. Inspired by the work of Bishop, in [25], the authors introduced a new version of Bishop frame by using a common vector field as binormal vector field of a regular curve and called this frame as "Type-2 Bishop frame". In 2008, Özdemir and Ergin extended Bishop frame to the non-lightlike curves in Minkowski 3-space, where they also called relative parallel adopted frame [18]. We know that the properties of geometric objects are independent of the choice of the coordinate systems. But, the researchers found that, when they adopted this frame, there will be some new geometric objects such as parallel slant curves, Bishop spherical images, Bishop Darboux images, etc.. After that, many regular curves and surfaces related to the Bishop frame have been treated in the Euclidean space [5, 6, 13-17, 22, 24], Minkowski space $[4,21,23,26]$, dual space [11] and Heisenberg group Heis ${ }^{3}$ [12]. The current study hopes to research those singular curves and surfaces associate to the Bishop frame instead of regular ones. We will classify the singularities of parallel lightcone developables, dual surface, Bishop pseudo-spherical Darboux images and Bishop pseudo-spherical images, which are generated by spacelike curves embedded

\footnotetext{
*Corresponding author

Email addresses: liuhm468@nenu.edu.cn (Haiming Liu), jiajing0407@126.com (Jiajing Miao), peidh340@nenu.edu.cn (Donghe Pei)

doi:10.22436/jnsa.010.09.39
} 
in Minkowski 3-space according to the relatively parallel adapted frame. Adopting the relatively parallel adapted frame as the basic tool, we get the main results of this paper, Proposition 2.2 and Theorem 2.3, which use the relatively parallel adapted frame for classification of singularity types of these geometric objects from the viewpoints of wave fronts and caustics. It is worth mentioning that we also investigate Legendrian dualities between pseudo-spherical images of spacelike curves in Minkowski 3-space by using the theory of Legendrian dualities (cf. Proposition 2.1) and get some meaningful properties of parallel slant helix (cf. Proposition 5.1).

The rest of this paper is organized as follows. Firstly, we introduce some basic concepts and the main results in the next section. Then, we introduce five different families of functions that will be useful to the study of geometric invariants of regular spacelike curves. Afterwards, some general results on the singularity theory are used for families of function germs and the main results are proved.

We shall assume throughout the whole paper that all the maps and manifolds are $C^{\infty}$ unless the contrary is explicitly stated.

\section{Basic concepts and results}

In this section, we introduce the basic notions and results in Lorentzian geometry. For more detail descriptions, see $[18,19]$. Let $\mathbb{R}_{1}^{3}$ denote the 3-dimensional Minkowski space, that is to say, the manifolds $\mathbb{R}^{3}$ with a flat Lorentzian metric $\langle$,$\rangle of signature (-,+,+)$, for any vectors $\mathbf{x}=\left(x_{1}, x_{2}, x_{3}\right)$ and $\mathbf{y}=\left(y_{1}, y_{2}, y_{3}\right)$ in $\mathbb{R}^{3},\langle\mathbf{x}, \mathbf{y}\rangle=-x_{1} y_{1}+x_{2} y_{2}+x_{3} y_{3}$. We also define a vector

$$
\mathbf{x} \wedge \mathbf{y}=\left|\begin{array}{ccc}
-\mathbf{e}_{1} & \mathbf{e}_{2} & \mathbf{e}_{3} \\
x_{1} & x_{2} & x_{3} \\
y_{1} & y_{2} & y_{3}
\end{array}\right|
$$

where $\left\{\mathbf{e}_{1}, \mathbf{e}_{2}, \mathbf{e}_{3}\right\}$ is the canonical basis of $\mathbb{R}_{1}^{3}$. One can easily show that $\langle\mathbf{x} \wedge \mathbf{y}, \mathbf{z}\rangle=\operatorname{det}(\mathbf{x}, \mathbf{y}, \mathbf{z})$. We say that a nonzero vector $\mathbf{x} \in \mathbb{R}_{1}^{3} \backslash\{\mathbf{0}\}$ is spacelike, lightlike or timelike if $\langle\mathbf{x}, \mathbf{x}\rangle>0,\langle\mathbf{x}, \mathbf{x}\rangle=0$ or $\langle\mathbf{x}, \mathbf{x}\rangle<0$ respectively. The norm of the vector $\mathbf{x} \in \mathbb{R}_{1}^{3}$ is defined by $\|\mathbf{x}\|=\sqrt{|\langle\mathbf{x}, \mathbf{x}\rangle|}$.

Let $\gamma: \mathrm{I} \rightarrow \mathbb{R}_{1}^{3}$ be a smooth regular curve (i.e., $\dot{\gamma}(\mathrm{t}) \neq 0$ for any $\mathrm{t} \in \mathrm{I}$ ), where $\mathrm{I}$ is an open interval. If $\langle\dot{\gamma}(\mathrm{t}), \dot{\gamma}(\mathrm{t})\rangle>0$ for any $\mathrm{t} \in \mathrm{I}$, we call such a curve a spacelike curve. The arc-length of a spacelike curve $\gamma(t)$, measured from $\gamma\left(t_{0}\right)$, is $s(t)=\int_{t_{0}}^{t}\|\dot{\gamma}(t)\| d t$. Then the parameter $s$ is determined such that $\left\|\gamma^{\prime}(s)\right\|=1$, where $\gamma^{\prime}(s)=\frac{d}{d s}$. So we say that a spacelike curve $\gamma$ is parameterized by arc-length if it satisfies that $\left\|\gamma^{\prime}(s)\right\|=1$. Throughout the remainder in this paper we denote the parameter s of $\gamma$ as the arc-length parameter. We denote $\mathbf{t}(s)=\gamma^{\prime}(s)$ and we call $\mathbf{t}(s)$ a unit tangent vector of $\gamma$ at $s$. We know that there exists an accompanying three-frames called Frenet frame for spacelike Frenet curve $\gamma(\mathrm{s})$. Denote by $\{\mathbf{T}(\mathrm{s}), \mathbf{N}(\mathrm{s}), \mathbf{B}(\mathrm{s})\}$ the moving Frenet frame along the unit speed spacelike Frenet curve $\gamma(\mathrm{s})$. Then, the Frenet formulas are given by

$$
\left(\begin{array}{c}
\mathbf{T}^{\prime}(s) \\
\mathbf{N}^{\prime}(s) \\
\mathbf{B}^{\prime}(s)
\end{array}\right)=\left(\begin{array}{ccc}
0 & k(s) & 0 \\
\delta(\mathbf{B}(s)) k(s) & 0 & \tau(s) \\
0 & \tau(s) & 0
\end{array}\right)\left(\begin{array}{c}
\mathbf{T}(s) \\
\mathbf{N}(s) \\
\mathbf{B}(s)
\end{array}\right) .
$$

Here, $\delta(\mathbf{x})=\langle\mathbf{x}, \mathbf{x}\rangle, k(s)$ and $\tau(s)$ are called curvature and torsion, respectively, see [15]. The Bishop frame equations for a parametrized unit length curve $\gamma(s)$ are as follows:

$$
\left(\begin{array}{c}
\mathbf{T}^{\prime}(s) \\
\mathbf{N}_{1}^{\prime}(s) \\
\mathbf{N}_{2}^{\prime}(s)
\end{array}\right)=\left(\begin{array}{ccc}
0 & k_{1}(s) & k_{2}(s) \\
-\delta\left(\mathbf{N}_{1}(s)\right) k_{1}(s) & 0 & 0 \\
-\delta\left(\mathbf{N}_{2}(s)\right) k_{2}(s) & 0 & 0
\end{array}\right)\left(\begin{array}{c}
\mathbf{T}(s) \\
\mathbf{N}_{1}(s) \\
\mathbf{N}_{2}(s)
\end{array}\right)
$$

Here, we will call the set $\left\{\mathbf{T}(\mathrm{s}), \mathbf{N}_{1}(\mathrm{~s}), \mathbf{N}_{2}(\mathrm{~s})\right\}$ as Bishop frame and $\mathrm{k}_{1}(\mathrm{~s})=\delta\left(\mathbf{N}_{1}(\mathrm{~s})\right)\left\langle\mathbf{T}^{\prime}(\mathrm{s}), \mathbf{N}_{1}(\mathrm{~s})\right\rangle$ and $\mathrm{k}_{2}(\mathrm{~s})=$ $\delta\left(\mathbf{N}_{2}(s)\right)\left\langle\mathbf{T}^{\prime}(\mathrm{s}), \mathbf{N}_{2}(\mathrm{~s})\right\rangle$ as Bishop curvatures. The relation matrix can be expressed as 


$$
\left(\begin{array}{c}
\mathbf{T}(s) \\
\mathbf{N}(s) \\
\mathbf{B}(s)
\end{array}\right)=\left(\begin{array}{ccc}
1 & 0 & 0 \\
0 & \cosh \theta(s) & \sinh \theta(s) \\
0 & \sinh \theta(s) & \cosh \theta(s)
\end{array}\right)\left(\begin{array}{c}
\mathbf{T}(s) \\
\mathbf{N}_{1}(s) \\
\mathbf{N}_{2}(s)
\end{array}\right)
$$

One can show that

$$
k(s)=\sqrt{\left|\delta\left(\mathbf{N}_{1}(s)\right) k_{1}^{2}(s)+\delta\left(\mathbf{N}_{2}(s)\right) k_{2}^{2}(s)\right|}, \quad \theta(s)=\operatorname{arctanh}\left(\frac{k_{2}(s)}{k_{1}(s)}\right),
$$

where $k_{1}(s) \neq 0, \tau(s)=\delta\left(\mathbf{N}_{1}(s)\right) \frac{d \theta(s)}{d s}$. Here, Bishop curvatures are also defined by

$$
\left\{\begin{array}{l}
k_{1}(s)=k(s) \cosh \theta(s), \\
k_{2}(s)=k(s) \sinh \theta(s),
\end{array}\right.
$$

and $\theta=\delta\left(\mathbf{N}_{1}(s)\right) \int \tau(s)$ ds. The orientation of the parallel transport frame includes the arbitrary choice of integration constant $\theta_{0}$, which disappears from $\tau$ (and hence from the Frenet frame) due to the differentiation [18]. Also, we assume that is positively oriented and the vector products of these vectors are defined as follows: $\mathbf{T}(\mathrm{s}) \wedge \mathbf{N}_{1}(\mathrm{~s})=\delta\left(\mathbf{N}_{2}(\mathrm{~s})\right) \mathbf{N}_{2}(\mathrm{~s}), \mathbf{N}_{1}(\mathrm{~s}) \wedge \mathbf{N}_{2}(\mathrm{~s})=\mathbf{T}(\mathrm{s}), \mathbf{N}_{2}(\mathrm{~s}) \wedge \mathbf{T}(\mathrm{s})=\delta\left(\mathbf{N}_{1}(\mathrm{~s})\right) \mathbf{N}_{1}(\mathrm{~s})$. We define the pseudo-spherical by

$$
\mathrm{Q}_{\epsilon}^{2}= \begin{cases}\mathrm{S}_{1}^{2}=\left\{\mathbf{x} \in \mathbb{R}_{1}^{3} \mid\langle\mathbf{x}, \mathbf{x}\rangle=1\right\}, & \text { if } \epsilon=+, \\ \mathrm{H}_{0}^{2}=\left\{\mathbf{x} \in \mathbb{R}_{1}^{3} \mid\langle\mathbf{x}, \mathbf{x}\rangle=-1\right\}, & \text { if } \epsilon=-.\end{cases}
$$

We call $S_{1}^{2}$ the de Sitter space and $\mathrm{H}_{0}^{2}$ the hyperbolic space. If we translate the three vector fields of Bishop trihedra to the center $\mathrm{O}$ of one of unit pseudo-spheres then we can get the tangent de Sitter indicatrix, the first Bishop pseudo-spherical indicatrix and the second Bishop pseudo-spherical indicatrix in [23] and we denote them by $\mathcal{T} \mathcal{D}(s)=\mathbf{T}(s), \mathcal{F} \mathcal{N}(s)=\mathbf{N}_{1}(s)$, and $\mathcal{S N}(s)=\mathbf{N}_{2}(s)$ separately. A lightlike unit circle is defined by

$$
S_{+}^{1}=\left\{\mathbf{x} \in \mathbb{R}_{1}^{3} \mid \mathbf{x}=\left(1, x_{2}, x_{3}\right), x_{2}^{2}+x_{3}^{2}=1\right\},
$$

and an open lightcone at the vertex $\mathbf{p}$ is defined by

$$
\mathrm{LC}_{\mathbf{p}}=\left\{\mathbf{x}=\left(\mathrm{x}_{1}, \mathrm{x}_{2}, \mathrm{x}_{3}\right) \in \mathbb{R}_{1}^{3} \mid-\left(\mathrm{x}_{1}-\mathrm{p}_{1}\right)^{2}+\left(\mathrm{x}_{2}-\mathrm{p}_{2}\right)^{2}+\left(\mathrm{x}_{3}-\mathrm{p}_{3}\right)^{2}=0\right\},
$$

where $\mathbf{p}=\left(p_{1}, p_{2}, p_{3}\right)$. For any lightlike vector $\mathbf{x}=\left(x_{1}, x_{2}, x_{3}\right)$, we write $\widetilde{\mathbf{x}}=\left(1, \frac{x_{2}}{x_{1}}, \frac{x_{3}}{x_{1}}\right) \in S_{+}^{1}$. Especially, we consider the following set on the lightcone and call it the lightcone bundle of curve $\gamma(\mathrm{s})$ through $\mathbf{v}_{0}$, for any $\mathbf{v}_{0} \in \mathbb{R}_{1}^{3}, \mathfrak{L} \mathfrak{C}\left(v_{0}\right)=\left\{\mathbf{u} \in \mathbb{R}_{1}^{3} \mid\left\langle\mathbf{u}-\mathbf{v}_{0}, \mathbf{u}-\mathbf{v}_{0}\right\rangle=0\right\}$.

Next, we will introduce a basic result on Legendrian dualities between pseudo-spheres [7] which has been proved to be a powerful tool for the study of surfaces in pseudo-spheres. One-forms on $\mathbb{R}_{1}^{3} \times \mathbb{R}_{1}^{3}$ are defined by $\langle\mathrm{d} \mathbf{v}, \mathbf{w}\rangle=-w_{0} \mathrm{~d} v_{0}+\sum_{i=1}^{2} w_{i} \mathrm{~d} v_{i}$ and $\langle\mathbf{v}, \mathrm{d} \mathbf{w}\rangle=-v_{0} \mathrm{~d} w_{0}+\sum_{i=1}^{2} v_{i} \mathrm{~d} w_{i}$. Then, we consider the following two double fibrations:

(1) (a) $\mathrm{H}_{0}^{2}(-1) \times \mathrm{S}_{1}^{2} \supset \Delta_{1}=\{(\mathbf{v}, \mathbf{w}) \mid\langle\mathbf{v}, \mathbf{w}\rangle=0\}$,

(b) $\pi_{11}: \Delta_{1} \longrightarrow \mathrm{H}_{0}^{2}(-1), \pi_{12}: \Delta_{1} \longrightarrow \mathrm{S}_{1}^{2}$

(c) $\theta_{11}=\left.\langle\mathrm{d} \mathbf{v}, \mathbf{w}\rangle\right|_{\Delta_{1}}, \theta_{12}=\left.\langle\mathbf{v}, \mathrm{d} \mathbf{w}\rangle\right|_{\Delta_{1}}$.

(2) (a) $S_{1}^{2} \times S_{1}^{2} \supset \Delta_{2}=\{(\mathbf{v}, \mathbf{w}) \mid\langle\mathbf{v}, \mathbf{w}\rangle=0\}$,

(b) $\pi_{21}: \Delta_{2} \longrightarrow S_{1}^{2}, \pi_{22}: \Delta_{2} \longrightarrow S_{1}^{2}$,

(c) $\theta_{21}=\left.\langle\mathrm{d} \mathbf{v}, \mathbf{w}\rangle\right|_{\Delta_{2}}, \theta_{22}=\left.\langle\mathbf{v}, \mathrm{d} \mathbf{w}\rangle\right|_{\Delta_{2}}$.

Here $\pi_{i 1}(\mathbf{v}, \mathbf{w})=\mathbf{v}, \pi_{i 2}(\mathbf{v}, \mathbf{w})=\mathbf{w}$. We remark that $\theta_{i 1}^{-1}(0)$ and $\theta_{i 2}^{-1}(0)$ define the same tangent plane field over $\Delta_{i}$ which is denoted by $K_{i}(i=1,2)$. The basic duality theorem is that each $\left(\Delta_{i}, K_{i}\right)$ is a contact manifold and both of $\pi_{i j}(j=1,2)$ are Legendrian fibrations. If there exists an isotropic mapping $i: L \longrightarrow \Delta_{i}$, which means that $i^{*} \theta_{i 1}=0$, we say that $\pi_{i 1}(i(L))$ and $\pi_{i 2}(i(L))$ are $\Delta_{i}$-dual to each other. It is easy to see that the condition $i^{*} \theta_{i 1}=0$ is equivalent to $i^{*} \theta_{i 2}=0$. Then we have the following proposition on the relationships between the first Bishop pseudo-spherical indicatrix and the second Bishop pseudospherical indicatrix with the help of the above Legendrian dualities. 
Proposition 2.1. Let $\gamma: \mathrm{I} \rightarrow \mathbb{R}_{1}^{3}$ be a unit speed spacelike curve. We have the following.

(1) $\mathbf{N}_{1}$ (s) and $\mathbf{N}_{2}$ (s) are $\Delta_{1}$-dual to each other.

(2) If $\delta(\mathbf{B}(s))=-1$, then $\mathbf{T}(\mathrm{s})$ and $\mathbf{B}(\mathrm{s})$ are $\Delta_{1}$-dual to each other, otherwise $\mathbf{T}(\mathrm{s})$ and $\mathbf{B}(\mathrm{s})$ are $\Delta_{2}$-dual to each other.

Proof.

(1) For the case that $\delta\left(\mathbf{N}_{1}(s)\right)=-1$, we consider the mapping

$$
\mathfrak{L}_{1}(\mathbf{s})=\left(\mathbf{N}_{1}(\mathrm{~s}), \mathbf{N}_{2}(\mathbf{s})\right),
$$

otherwise we change the positions of $\mathbf{N}_{1}(s)$ and $\mathbf{N}_{2}(s)$ in the mapping $\mathfrak{L}_{1}(\mathrm{~s})$. Then we have $\left\langle\mathbf{N}_{1}(s), \mathbf{N}_{2}(s)\right\rangle=$ 0 and

$$
\mathfrak{L}_{1}^{*} \theta_{12}(s)=\left\langle\mathbf{N}_{1}(s), \mathbf{N}_{2}^{\prime}(s)\right\rangle=\left\langle\mathbf{N}_{1}(s),-\delta\left(\mathbf{N}_{2}(s)\right) k_{2}(s) \mathbf{T}(s)\right\rangle=0 .
$$

The assertion (1) follows.

(2) For the case that $\delta(\mathbf{B}(s))=-1$, we consider the mapping

$$
\mathfrak{L}_{2}(\mathrm{~s})=(\mathbf{B}(\mathrm{s}), \mathbf{T}(\mathrm{s})) .
$$

Then we have $\langle\mathbf{B}(\mathrm{s}), \mathbf{T}(\mathrm{s})\rangle=0$ and

$$
\mathfrak{L}_{2}^{*} \theta_{11}(s)=\left\langle\mathbf{B}^{\prime}(s), \mathbf{T}(s)\right\rangle=\langle\tau(s) \mathbf{N}(s), \mathbf{T}(s)\rangle=0 .
$$

The first claim of assertion (2) follows. Using the same computation as the above proof, we can get the second claim.

We define the parallel lightcone developables of $\gamma(\mathrm{s})$ as the map $\mathcal{P} \mathcal{L} \mathcal{D}_{\gamma}^{ \pm}: \mathrm{I} \times \mathbb{R} \rightarrow \mathbb{R}_{1}^{3}$ given by

$$
\mathcal{P} \mathcal{L} \mathcal{D}_{\gamma}^{ \pm}(s, u)=\gamma(s)+u\left(\mathbf{N}_{1}(s) \pm \mathbf{N}_{2}(s)\right)
$$

If $\delta\left(\mathbf{N}_{1}(s)\right) k_{1}(s) \pm \delta\left(\mathbf{N}_{2}(s)\right) k_{2}(s) \neq 0$ and $k_{1}(s) \neq 0$, we can define

$$
\mathbf{d}_{\epsilon}(s)=\frac{\left|k_{1}(s)\right|}{\sqrt{\left|k_{1}^{2}(s)-k_{2}^{2}(s)\right|}}\left(\frac{k_{2}(s)}{k_{1}(s)} \mathbf{N}_{1}(s)+\mathbf{N}_{2}(s)\right) .
$$

We call every $\mathbf{d}_{ \pm}(\mathbf{s})$ the pseudo-spherical Darboux images of $\gamma(\mathrm{s})$. The dual surface of $\gamma$ is defined by

$$
\mathcal{B D U}_{\gamma}(\mathbf{v}, \mathrm{u})=\left\{(\mathbf{v}, \mathrm{u}) \in \mathrm{Q}_{\epsilon}^{2} \times \mathbb{R} \mid \mathrm{u}=\langle\gamma(\mathrm{s}), \mathbf{v}\rangle,\langle\mathbf{T}(\mathrm{s}), \mathbf{v}\rangle=0\right\}
$$

We define a function $\rho(s)=k_{1}^{\prime}(s) k_{2}(s)-k_{1}(s) k_{2}^{\prime}(s)$ of a regular spacelike curve in $\mathbb{R}_{1}^{3}$ and we can describe some properties of parallel slant curve by $\rho(s)$. Let $F: \mathbb{R}_{1}^{3} \rightarrow \mathbb{R}$ be a submersion and $\gamma: \mathrm{I} \rightarrow \mathbb{R}_{1}^{3}$ be a regular unit speed spacelike Frenet curve. We say that $\gamma(s)$ and $F^{-1}(0)$ have k-point contact for $s=s_{0}$ if the function $\mathrm{g}(\mathrm{s})=\mathrm{F} \circ \gamma(\mathrm{s})$ satisfies

$$
g\left(s_{0}\right)=g^{\prime}\left(s_{0}\right)=g^{\prime \prime}\left(s_{0}\right)=\cdots=g^{(k-1)}\left(s_{0}\right)=0, g^{(k)}\left(s_{0}\right) \neq 0 .
$$

We also say that $\gamma(\mathrm{s})$ and $\mathrm{F}^{-1}(0)$ have at least k-point contact for $\mathrm{s}=\mathrm{s}_{0}$ if the function $\mathrm{g}(\mathrm{s})=\mathrm{F} \circ \gamma(\mathrm{s})$ satisfies $g\left(s_{0}\right)=g^{\prime}\left(s_{0}\right)=g^{\prime \prime}\left(s_{0}\right)=\cdots=g^{(k-1)}\left(s_{0}\right)=0$. Let function germ $F:\left(\mathbb{R} \times \mathbb{R}^{r},(s, x)\right) \rightarrow \mathbb{R}$ be an $r$-parameter unfolding of $f(s)$, where $f(s)=F\left(s, x_{0}\right)$. We say that $f(s)$ has $A_{k}$-singularity at $s_{0}$ if $f^{(p)}\left(s_{0}\right)=0$ for all $1 \leqslant p \leqslant k$, and $f^{(k+1)}\left(s_{0}\right) \neq 0$. We also say that $f(s)$ has $A_{k}$-singularity at $s_{0}$ if $f^{(p)}\left(s_{0}\right)=0$ for all $1 \leqslant p \leqslant k$. We give a brief review on Legendrian singularity theory mainly due to Arnold [1]. The main 
tool of Legendrian singularities theory is the notion of generating families. Let $G:\left(\mathbb{R} \times \mathbb{R}^{n}, \mathbf{0}\right) \rightarrow(\mathbb{R}, 0)$ be a function germ. We say that $G$ is a Morse family if the mapping

$$
\Delta^{*} \mathrm{G}=\left(\mathrm{G}, \frac{\partial \mathrm{G}}{\partial \mathrm{s}}\right):\left(\mathbb{R} \times \mathbb{R}^{\mathrm{n}}, \mathbf{0}\right) \longrightarrow(\mathbb{R} \times \mathbb{R}, \mathbf{0})
$$

is non-singular, where $(s, \mathbf{v})=\left(s, v_{1}, \ldots, v_{n}\right) \in\left(\mathbb{R} \times \mathbb{R}^{n}, \mathbf{0}\right)$. In this case we have a smooth $n$-1-dimensional submanifold,

$$
\Sigma_{*}(G)=\left\{(s, \mathbf{v}) \in\left(\mathbb{R} \times \mathbb{R}^{n}, \mathbf{0}\right) \mid G(s, \mathbf{v})=\frac{\partial G}{\partial s}(s, \mathbf{v})=0\right\}
$$

and the map germ $\Phi_{\mathrm{G}}:\left(\Sigma_{*}(\mathrm{G}), \mathbf{0}\right) \longrightarrow \mathrm{PT}^{*} \mathbb{R}^{\mathrm{n}}$ defined by

$$
\Phi_{\mathrm{G}}(\mathrm{s}, \mathbf{v})=\left(\mathbf{v},\left[\frac{\partial \mathrm{G}}{\partial v_{1}}(\mathrm{~s}, \mathbf{v}): \ldots: \frac{\partial \mathrm{G}}{\partial v_{\mathrm{n}}}(\mathrm{s}, \mathbf{v})\right]\right)
$$

is a Legendrian immersion germ. We call $\mathrm{G}$ a generating family of $\Phi_{\mathrm{G}}\left(\Sigma_{*} \mathrm{G}\right)$. Therefore the corresponding wave front is

$$
W\left(\Phi_{G}\right)=\left\{\mathbf{v} \in \mathbb{R}^{n} \mid \exists s \in \mathbb{R} \text { such that } G(s, \mathbf{v})=\frac{\partial G}{\partial s}(s, \mathbf{v})=0\right\} .
$$

We sometimes denote $\mathrm{D}_{\mathrm{G}}=\mathrm{W}\left(\Phi_{\mathrm{G}}\right)$ and call it the discriminant set of $\mathrm{G}$.

Now, we can apply the above arguments to our situation and we get the following proposition which indicates that parallel lightcone developables, dual surface and Bishop pseudo-spherical images of spacelike curves can be seen as wave fronts which have Legendrian singularities in the framework of the theory of Legendrian singularity and Bishop pseudo-spherical Darboux images can be seen as caustics which have Lagrangian singularities in the framework of the theory of Lagrangian singularity (see [1] for details). The main results of this paper are contained in the following proposition and theorem.

Proposition 2.2. The parallel developables and dual surface are two dimensional wave fronts which have $A_{1^{-}}$ singularity, $A_{2}$-singularity, and $A_{3}$-singularity. Bishop pseudo-spherical images are one dimensional wave fronts which have $A_{1}$-singularity and $A_{2}$-singularity. Bishop pseudo-spherical Darboux images can be seen as one dimensional caustics which have $\mathrm{A}_{2}$-singularity and $\mathrm{A}_{3}$-singularity.

The following theorem gives some more detail descriptions on the conditions of those singularities.

Theorem 2.3. Let $\gamma: \mathrm{I} \rightarrow \mathbb{R}_{1}^{3}$ be a unit speed regular spacelike curve, we have the following.

(A) For $\mathbf{v}_{0}=\mathfrak{P} \mathfrak{L} \mathfrak{D}^{ \pm}\left(\mathrm{s}_{0}, \mu_{0}\right)$ and the lightcone bundle $\mathfrak{L} \mathfrak{C}\left(v_{0}\right)$ of the curve, suppose that

$$
\delta\left(\mathbf{N}_{1}(s)\right) k_{1}(s) \pm \delta\left(\mathbf{N}_{2}(s)\right) k_{2}(s) \neq 0 .
$$

Then, one has the following claims.

(1) The curve $\gamma(\mathrm{s})$ and $\mathfrak{L C}\left(v_{0}\right)$ have at least 2-point contact for $\mathrm{s}_{0}$.

(2) The curve $\gamma(\mathrm{s})$ and $\mathfrak{L C}\left(v_{0}\right)$ have at least 3-point contact for $\mathrm{s}_{0}$ if and only if

$$
\mathbf{v}_{0}=\gamma\left(s_{0}\right)+\frac{1}{\delta\left(\mathbf{N}_{1}\left(s_{0}\right)\right) k_{1}\left(s_{0}\right) \pm \delta\left(\mathbf{N}_{2}\left(s_{0}\right)\right) k_{2}\left(s_{0}\right)}\left(\mathbf{N}_{1}\left(s_{0}\right) \pm \mathbf{N}_{2}\left(s_{0}\right)\right)
$$

and $\delta\left(\mathbf{N}_{1}\left(s_{0}\right)\right) k_{1}^{\prime}\left(s_{0}\right) \pm \delta\left(\mathbf{N}_{2}\left(s_{0}\right)\right) k_{2}^{\prime}\left(s_{0}\right) \neq 0$. Under this condition, the germ of image $\mathfrak{P} \mathfrak{L} \mathfrak{D}^{ \pm}(\mathrm{s}, \mu)$ at $\mathfrak{P} \mathfrak{L} \mathfrak{D}^{ \pm}\left(s_{0}, \mu_{0}\right)$ is locally diffeomorphic to the cuspidal edge $\mathrm{C} \times \mathbb{R}$ and the locus of its singularity points $\mathfrak{P} \mathfrak{L} \mathfrak{D}^{ \pm}\left(s_{0}, \mu_{0}\right)$ is locally diffeomorphic to the line (cf., Figure 1$)$, where

$$
\mu_{0}=\frac{-1}{\delta\left(\mathbf{N}_{1}\left(s_{0}\right)\right) k_{1}\left(s_{0}\right) \pm \delta\left(\mathbf{N}_{2}\left(s_{0}\right)\right) k_{2}\left(s_{0}\right)} .
$$

(3) The curve $\gamma(\mathrm{s})$ and $\mathfrak{L C}\left(v_{0}\right)$ have at least 4-point contact for $\mathrm{s}_{0}$ if and only if

$$
\begin{aligned}
& \mathbf{v}_{0}=\gamma\left(s_{0}\right)+\frac{1}{\delta\left(\mathbf{N}_{1}\left(s_{0}\right)\right) k_{1}\left(s_{0}\right) \pm \delta\left(\mathbf{N}_{2}\left(s_{0}\right)\right) k_{2}\left(s_{0}\right)}\left(\mathbf{N}_{1}\left(s_{0}\right) \pm \mathbf{N}_{2}\left(s_{0}\right)\right), \\
& \delta\left(\mathbf{N}_{1}\left(s_{0}\right)\right) k_{1}^{\prime}\left(s_{0}\right) \pm \delta\left(\mathbf{N}_{2}\left(s_{0}\right)\right) k_{2}^{\prime}\left(s_{0}\right)=0 \text { and }
\end{aligned}
$$




$$
\left(k_{1}\left(s_{0}\right) \mp k_{2}\left(s_{0}\right)\right)^{2}+\left(k_{1}\left(s_{0}\right) \mp k_{2}\left(s_{0}\right)\right)\left(k_{2}^{2}\left(s_{0}\right)-k_{1}^{2}\left(s_{0}\right)\right)-\delta\left(\mathbf{N}_{1}\left(s_{0}\right)\right) k_{1}^{\prime \prime}\left(s_{0}\right) \mp \delta\left(\mathbf{N}_{2}\left(s_{0}\right)\right) k_{2}^{\prime \prime}\left(s_{0}\right) \neq 0 .
$$

Under this condition, the germ of image $\mathfrak{P} \mathfrak{L D}^{ \pm}(s, \mu)$ at $\mathfrak{P} \mathfrak{L} \mathfrak{D}^{ \pm}\left(s_{0}, \mu_{0}\right)$ is locally diffeomorphic to the swallowtail and the locus of its singularity points $\mathfrak{P} \mathfrak{L D}^{ \pm}\left(\mathrm{s}_{0}, \frac{-1}{\left.\delta\left(\mathbf{N}_{1}\left(\mathbf{s}_{0}\right)\right) \mathrm{k}_{1}\left(\mathrm{~s}_{0}\right) \pm \delta\left(\mathbf{N}_{2}\left(\mathbf{s}_{0}\right)\right) \mathrm{k}_{2}\left(\mathbf{s}_{0}\right)\right)}\right)$ is locally diffeomorphic to the $(2,3,4)$-cusp (cf., Figure 2).

(B) Suppose that $\delta\left(\mathbf{N}_{1}(s)\right) k_{1}(s) \pm \delta\left(\mathbf{N}_{2}(s)\right) k_{2}(s) \neq 0$ and $k_{1}(s) \neq 0$. Then, one have the following claims.

(1) The Bishop dual $\mathcal{B D U}_{\gamma}$ is locally diffeomorphic to the cuspidaledge $\mathrm{C} \times \mathbb{R}$ at $\mathrm{s}_{0}$ if

$$
\mathbf{v}= \pm \frac{\left|k_{1}\left(s_{0}\right)\right|}{\sqrt{\left|k_{1}^{2}\left(s_{0}\right)-k_{2}^{2}\left(s_{0}\right)\right|}}\left(\frac{k_{2}\left(s_{0}\right)}{k_{1}\left(s_{0}\right)} \mathbf{N}_{1}\left(s_{0}\right)+\mathbf{N}_{2}\left(s_{0}\right)\right)
$$

$\mathrm{u}=\left\langle\gamma\left(\mathrm{s}_{0}\right), \mathbf{v}\right\rangle$ and $\rho\left(\mathrm{s}_{0}\right) \neq 0$. Under this condition, the pseudo-spherical Darboux image of spacelike curve is locally diffeomorphic to a line $\{0\} \times \mathbb{R}$ at $\mathrm{s}_{0}$ (cf., Figure 1 ).

(2) The Bishop dual $\mathcal{B D U}_{\gamma}$ is locally diffeomorphic to the $\mathrm{SW}$ at $\mathrm{s}_{0}$ if

$$
\mathbf{v}= \pm \frac{\left|k_{1}\left(s_{0}\right)\right|}{\sqrt{\left|k_{1}^{2}\left(s_{0}\right)-k_{2}^{2}\left(s_{0}\right)\right|}}\left(\frac{k_{2}\left(s_{0}\right)}{k_{1}\left(s_{0}\right)} \mathbf{N}_{1}\left(s_{0}\right)+\mathbf{N}_{2}\left(s_{0}\right)\right),
$$

$u=\left\langle\gamma\left(s_{0}\right), \mathbf{v}\right\rangle, \rho\left(s_{0}\right)=0$ and $\rho^{\prime}\left(s_{0}\right) \neq 0$. Under this condition, the pseudo-spherical Darboux image of spacelike curve is locally diffeomorphic to the cusp $\mathrm{C}$ at $\mathrm{s}_{0}$ (cf., Figures 2 and 3).

(C) Suppose that $k_{2}(s) \neq 0$. Then, one has the following claims.

(1) The first Bishop pseudo-spherical image $\mathbf{N}_{1}(\mathrm{~s})$ is locally diffeomorphic to a line $\{0\} \times \mathbb{R}$ at $s_{0}$ if $k_{1}\left(s_{0}\right) \neq 0$.

(2) The first Bishop pseudo-spherical image $\mathbf{N}_{1}(\mathrm{~s})$ is locally diffeomorphic to the cusp $\mathrm{C}$ at $\mathrm{s}_{0}$ if $\mathrm{k}_{1}\left(\mathrm{~s}_{0}\right)=0$ and $k_{1}^{\prime}\left(s_{0}\right) \neq 0$ (cf., Figure 3 ).

(D) Suppose that $\mathrm{k}_{1}(\mathrm{~s}) \neq 0$. Then, one has the following claims.

(1) The second Bishop pseudo-spherical image $\mathbf{N}_{2}(s)$ is locally diffeomorphic to a line $\{0\} \times \mathbb{R}$ at $s_{0}$ if $k_{2}\left(s_{0}\right) \neq 0$.

(2) The second Bishop pseudo-spherical image $\mathbf{N}_{2}(\mathrm{~s})$ is locally diffeomorphic to the cusp C at $\mathrm{s}_{0}$ if $\mathrm{k}_{2}\left(\mathrm{~s}_{0}\right)=0$ and $\mathrm{k}_{2}^{\prime}\left(\mathrm{s}_{0}\right) \neq 0$ (cf., Figure 3 ).

Here, cusp is $C=\left\{\left(x_{1}, x_{2}\right) \mid x_{1}^{2}=x_{2}^{3}\right\}$, cuspidal edge is $C \times \mathbb{R}=\left\{\left(x_{1}, x_{2}\right) \mid x_{1}^{2}=x_{2}^{3}\right\} \times \mathbb{R}$, swallowtail is

$$
\mathrm{SW}=\left\{\left(x_{1}, x_{2}, x_{3}\right) \mid x_{1}=3 u^{4}+u^{2} v, x_{2}=4 u^{3}+2 u v, x_{3}=v\right\},
$$

and $(2,3,4)$-cusp is $\mathrm{C}(2,3,4)=\left\{\left(\mathrm{t}^{2}, \mathrm{t}^{3}, \mathrm{t}^{4}\right) \in \mathbb{R}^{3} \mid \mathrm{t} \in \mathbb{R}\right\}$. The pictures of cuspidal edge, swallowtail, and cusp will be seen in Figures 1, 2, and 3, respectively.

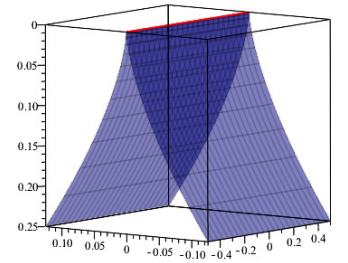

Figure 1: Cuspidal edge.

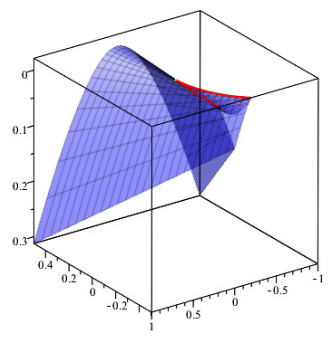

Figure 2: Swallowtail.

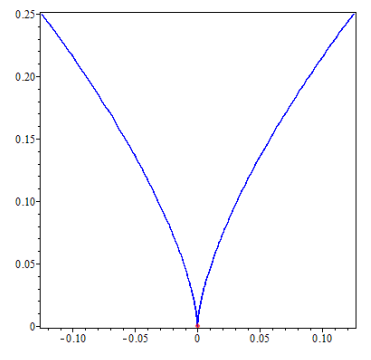

Figure 3: Cusp.

\section{Functions on spacelike curve which can be seen as generating families}

The purpose of this section is to construct some functions which can be seen as generating families and these functions will be useful to prove the main results. 
We need to use some general results on the singularity theory for families of function germs. Detailed descriptions can be found in the book [3]. Let $F(s, x)$ be an unfolding of $f(s)$ and $f(s)$ has $A_{k}$-singularity $(k \geqslant 1)$ at $s_{0}$. We denote the $(k-1)$-jet of the partial derivative $\frac{\partial F}{\partial x_{i}}\left(s, x_{0}\right)$ at $s_{0}$ by

$$
j^{(k-1)}\left(\frac{\partial F}{\partial x_{i}}\left(s, x_{0}\right)\right)\left(s_{0}\right)=\sum_{j=1}^{k-1} a_{j i} s^{j}
$$

for $i=1, \ldots, r$. Then, $F(s, x)$ is called an $\mathcal{R}$-versal unfolding if the $k \times r$ matrix of coefficients $\left(a_{0 i}, a_{j i}\right)$ has rank $k,(k \leqslant r)$, where $a_{0 i}=\frac{\partial F}{\partial x_{i}}\left(s_{0}, x_{0}\right) . F(s, x)$ is called an $\mathcal{R}^{+}$-versal unfolding, if the $(k-1) \times r$ matrix of coefficients $\left(a_{j i}\right)$ has rank $k-1,(k-1 \leqslant r)$. We now introduce the other important set concerning unfoldings. The bifurcation set of an $\mathcal{R}^{+}$-versal unfolding $\mathrm{F}(\mathrm{s}, \mathbf{x})$ is the set

$$
\mathfrak{B}_{\mathrm{F}}=\left\{\mathbf{x} \in \mathbb{R}^{\mathrm{r}} \mid \frac{\partial \mathrm{F}}{\partial s}(s, \mathbf{x})=\frac{\partial^{2} \mathrm{~F}}{\partial s^{2}}(s, \mathbf{x})=0\right\}
$$

and it can be seen as caustic from the viewpoint of Lagrangian singularity theory. To prove the main results in this paper, we need the following well-known result ([3]).

Theorem 3.1. Let $\mathrm{F}:\left(\mathbb{R} \times \mathbb{R}^{\mathrm{r}},\left(\mathrm{s}_{0}, \mathbf{x}_{0}\right)\right) \longrightarrow \mathbb{R}$ be an r-parameter unfolding of $\mathrm{f}(\mathrm{s})$ which has the $\mathrm{A}_{\mathrm{k}}$ singularity at $\mathrm{s}_{0}$.

(A) Suppose that $\mathrm{F}(\mathrm{s}, \mathbf{x})$ is an $\mathcal{R}$-versal unfolding, then we have the following:

(1) if $\mathrm{k}=1$, then $\mathfrak{D}_{\mathrm{F}}$ is locally diffeomorphic to $0 \times \mathbb{R}^{\mathrm{r}-1}$;

(2) if $\mathrm{k}=2$, then $\mathfrak{D}_{\mathrm{F}}$ is locally diffeomorphic to $\mathrm{C} \times \mathbb{R}^{\mathrm{r}-2}$;

(3) if $\mathrm{k}=3$, then $\mathfrak{D}_{\mathrm{F}}$ is locally diffeomorphic to $\mathrm{SW} \times \mathbb{R}^{\mathrm{r}-3}$.

(B) Suppose that $\mathrm{F}(\mathrm{s}, \mathbf{x})$ is an $\mathcal{R}^{+}$-versal unfolding, then we have the following:

(1) if $\mathrm{k}=2$, then $\mathfrak{B}_{\mathrm{F}}$ is locally diffeomorphic to $0 \times \mathbb{R}^{\mathrm{r}-1}$;

(2) if $\mathrm{k}=3$, then $\mathfrak{B}_{\mathrm{F}}$ is locally diffeomorphic to $\mathrm{C} \times \mathbb{R}^{\mathrm{r}-2}$.

We will apply the above unfolding theory of function germ to the following five different families of functions on a spacelike curve to prove Proposition 2.2 and Theorem 2.3.

Let $\gamma: \mathrm{I} \rightarrow \mathbb{R}_{1}^{3}$ be a unit speed regular spacelike curve. We define a function

$$
\mathrm{G}: \mathrm{I} \times \mathbb{R}_{1}^{3} \rightarrow \mathbb{R} \text {, by } \mathrm{G}(\mathrm{s}, \mathbf{v})=\langle\gamma(\mathrm{s})-\mathbf{v}, \gamma(\mathrm{s})-\mathbf{v}\rangle \text {. }
$$

We call $G$ the Lorentzian distance-square function on a spacelike curve $\gamma$. For any fixed vector $\mathbf{v} \in \mathbb{R}_{1}^{3}$, we denote $g_{v}(s)=G(s, \mathbf{v})$. We also define two families of smooth functions

$$
\mathrm{H}: \mathrm{I} \times \mathrm{Q}_{\epsilon}^{2} \rightarrow \mathbb{R} \text { by } \mathrm{H}(\mathrm{s}, \mathbf{v})=\langle\gamma(\mathrm{s}), \mathbf{v}\rangle
$$

and

$$
\widetilde{\mathrm{H}}: \mathrm{I} \times \mathrm{Q}_{\epsilon}^{2} \times \mathbb{R} \longrightarrow \mathbb{R} \text { by } \widetilde{\mathrm{H}}(\mathrm{s}, \mathbf{v}, \mathrm{u})=\mathrm{H}(\mathrm{s}, \mathbf{v})-\mathrm{u}=\langle\gamma(\mathrm{s}), \mathbf{v}\rangle-\mathrm{u} .
$$

We call each of them height function and extended height function of $\gamma$, respectively. For any fixed $\mathbf{v} \in \mathrm{Q}_{\epsilon}^{2}$, we denote $h_{v}(s)=H(s, v)$ and $\widetilde{h}_{v}(s)=\widetilde{H}(s, \mathbf{v})$. We define two families of smooth functions on I as follows:

$$
\mathrm{H}_{\mathrm{i}}: \mathrm{I} \times \mathrm{Q}_{\epsilon}^{2} \rightarrow \mathbb{R} \text { by } \mathrm{H}_{\mathrm{i}}(\mathrm{s}, \mathbf{v})=\left\langle\mathbf{N}_{\mathrm{i}}(\mathrm{s}), \mathbf{v}\right\rangle,
$$

where $i=1,2$. We call it the first (resp. second) Bishop normal indicatrix height function when $i=1$ (resp. $i=2)$. For any fixed $\mathbf{v} \in Q_{\epsilon}^{2}$, we denote $h_{i v}(s)=H_{i}(s, \mathbf{v})$. We have the following proposition.

Proposition 3.2. Let $\gamma: \mathrm{I} \rightarrow \mathbb{R}_{1}^{3}$ be a unit speed regular spacelike curve.

(A) Suppose $\delta\left(\mathbf{N}_{1}(\mathrm{~s})\right) \mathrm{k}_{1}(\mathrm{~s}) \pm \delta\left(\mathbf{N}_{2}(\mathrm{~s})\right) \mathrm{k}_{2}(\mathrm{~s}) \neq 0$, then we have the following. 
(1) $g_{v}(s)=g_{v}^{\prime}(s)=0$ if and only if

$$
\gamma(\mathrm{s})-\mathbf{v}=\lambda\left(\mathbf{N}_{1}(\mathrm{~s}) \pm \mathbf{N}_{2}(\mathrm{~s})\right), \lambda \in \mathbb{R} \backslash\{0\} ;
$$

(2) $g_{v}(s)=g_{v}^{\prime}(s)=g_{v}^{\prime \prime}(s)=0$ if and only if

$$
\gamma(\mathrm{s})-\mathbf{v}=\frac{-1}{\delta\left(\mathbf{N}_{1}(\mathrm{~s})\right) \mathrm{k}_{1}(\mathrm{~s}) \pm \delta\left(\mathbf{N}_{2}(\mathrm{~s})\right) \mathrm{k}_{2}(\mathrm{~s})}\left(\mathbf{N}_{1}(\mathrm{~s}) \pm \mathbf{N}_{2}(\mathrm{~s})\right)
$$

and $\delta\left(\mathbf{N}_{1}(s)\right) k_{1}(s) \pm \delta\left(\mathbf{N}_{2}(s)\right) k_{2}(s) \neq 0$;

(3) $g_{v}(s)=g_{v}^{\prime}(s)=g_{v}^{\prime \prime}(s)=g_{v}^{(3)}(s)=0$ if and only if

$$
\gamma(s)-\mathbf{v}=\frac{-1}{\delta\left(\mathbf{N}_{1}(s)\right) k_{1}(s) \pm \delta\left(\mathbf{N}_{2}(s)\right) k_{2}(s)}\left(\mathbf{N}_{1}(s) \pm \mathbf{N}_{2}(s)\right)
$$

and

$$
\delta\left(\mathbf{N}_{1}(\mathrm{~s})\right) k_{1}^{\prime}(\mathrm{s}) \pm \delta\left(\mathbf{N}_{2}(\mathrm{~s})\right) k_{2}^{\prime}(\mathrm{s})=0 ;
$$

(4) $g_{v}(s)=g_{v}^{\prime}(s)=g_{v}^{\prime \prime}(s)=g_{v}^{(3)}(s)=g_{v}^{(4)}(s)=0$ if and only if

$$
\begin{gathered}
\gamma(s)-\mathbf{v}=\frac{-1}{\delta\left(\mathbf{N}_{1}(s)\right) k_{1}(s) \pm \delta\left(\mathbf{N}_{2}(s)\right) k_{2}(s)}\left(\mathbf{N}_{1}(s) \pm \mathbf{N}_{2}(s)\right), \\
\delta\left(\mathbf{N}_{1}(s)\right) k_{1}^{\prime}(s) \pm \delta\left(\mathbf{N}_{2}(s)\right) k_{2}^{\prime}(s)=0 \text { and } \\
\left(k_{1}(s) \mp k_{2}(s)\right)^{2}+\left(k_{1}(s) \mp k_{2}(s)\right)\left(k_{2}^{2}(s)-k_{1}^{2}(s)\right)-\delta\left(\mathbf{N}_{1}(s)\right) k_{1}^{\prime \prime}(s) \mp \delta\left(\mathbf{N}_{2}(s)\right) k_{2}^{\prime \prime}(s)=0 .
\end{gathered}
$$

(B) Suppose that $\delta\left(\mathbf{N}_{1}(s)\right) k_{1}(s) \pm \delta\left(\mathbf{N}_{2}(s)\right) k_{2}(s) \neq 0$ and $k_{1}(s) \neq 0$. Then we have the following.

(1) $h_{v}^{\prime}(s)=0$ if and only if there are real numbers $\lambda$ and $\mu$ such that $\mathbf{v}=\lambda \mathbf{N}_{1}(s)+\mu \mathbf{N}_{2}$ (s) and

$$
\delta\left(\mathbf{N}_{1}(s)\right) \lambda^{2}+\delta\left(\mathbf{N}_{2}(s)\right) \mu^{2}= \pm 1
$$

(2) $h_{v}^{\prime}(s)=h_{v}^{\prime \prime}(s)=0$ if and only if

$$
\mathbf{v}= \pm \frac{\left|k_{1}(s)\right|}{\sqrt{\left|k_{1}^{2}(s)-k_{2}^{2}(s)\right|}}\left(\frac{k_{2}(s)}{k_{1}(s)} \mathbf{N}_{1}(s)+\mathbf{N}_{2}(s)\right)
$$

(3) $h_{v}^{\prime}(s)=h_{v}^{\prime \prime}(s)=h_{v}^{(3)}(s)=0$ if and only if

$$
\mathbf{v}= \pm \frac{\left|k_{1}(s)\right|}{\sqrt{\left|k_{1}^{2}(s)-k_{2}^{2}(s)\right|}}\left(\frac{k_{2}(s)}{k_{1}(s)} \mathbf{N}_{1}(s)+\mathbf{N}_{2}(s)\right)
$$

and

$$
k_{1}^{\prime}(s) k_{2}(s)-k_{1}(s) k_{2}^{\prime}(s)=0 ;
$$

(4) $h_{v}^{\prime}(s)=h_{v}^{\prime \prime}(s)=h_{v}^{(3)}(s)=h_{v}^{(4)}(s)=0$ if and only if

$$
\mathbf{v}= \pm \frac{\left|k_{1}(s)\right|}{\sqrt{\left|k_{1}^{2}(s)-k_{2}^{2}(s)\right|}}\left(\frac{k_{2}(s)}{k_{1}(s)} \mathbf{N}_{1}(s)+\mathbf{N}_{2}(s)\right)
$$

and

$$
k_{1}^{\prime}(s) k_{2}(s)-k_{1}(s) k_{2}^{\prime}(s)=\left(k_{1}^{\prime}(s) k_{2}(s)-k_{1}(s) k_{2}^{\prime}(s)\right)^{\prime}=0 .
$$

(C) Suppose that $\delta\left(\mathbf{N}_{1}(\mathrm{~s})\right) \mathrm{k}_{1}(\mathrm{~s}) \pm \delta\left(\mathbf{N}_{2}(\mathrm{~s})\right) \mathrm{k}_{2}(\mathrm{~s}) \neq 0$ and $\mathrm{k}_{1}(\mathrm{~s}) \neq 0$. Then we have the following. 
(1) $\widetilde{\mathrm{h}}_{v}(\mathrm{~s})=0$ if and only if $\mathrm{u}=\langle\gamma(\mathrm{s}), \mathbf{v}\rangle$;

(2) $\widetilde{\mathrm{h}}_{v}(\mathrm{~s})=\widetilde{\mathrm{h}}_{v}^{\prime}(\mathrm{s})=0$ if and only if there are real numbers $\lambda$ and $\mu$ such that $\mathbf{v}=\lambda \mathbf{N}_{1}(\mathrm{~s})+\mu \mathbf{N}_{2}(\mathrm{~s})$, $\delta\left(\mathbf{N}_{1}(s)\right) \widetilde{\sim}^{2}+\delta\left(\mathbf{N}_{2}(s)\right) \mu^{2}= \pm 1$ and $\mathbf{u}=\langle\gamma(s), \mathbf{v}\rangle$;

(3) $\widetilde{h}_{v}(s)=\widetilde{h}_{v}^{\prime}(s)=\widetilde{h}_{v}^{\prime \prime}(s)=0$ if and only if

$$
\mathbf{v}= \pm \frac{\left|k_{1}(s)\right|}{\sqrt{\left|k_{1}^{2}(s)-k_{2}^{2}(s)\right|}}\left(\frac{k_{2}(s)}{k_{1}(s)} \mathbf{N}_{1}(s)+\mathbf{N}_{2}(s)\right)
$$

and $\mathbf{u}=\langle\gamma(\mathrm{s}), \mathbf{v}\rangle$;

(4) $\widetilde{\mathrm{h}}_{v}(\mathrm{~s})=\widetilde{\mathrm{h}}_{v}^{\prime}(\mathrm{s})=\widetilde{\mathrm{h}}_{v}^{\prime \prime}(\mathrm{s})=\widetilde{\mathrm{h}}_{v}^{(3)}(\mathrm{s})=0$ if and only if

$$
\mathbf{v}= \pm \frac{\left|k_{1}(s)\right|}{\sqrt{\left|k_{1}^{2}(s)-k_{2}^{2}(s)\right|}}\left(\frac{k_{2}(s)}{k_{1}(s)} \mathbf{N}_{1}(s)+\mathbf{N}_{2}(s)\right)
$$

$u=\langle\gamma(s), v\rangle$, and $k_{1}^{\prime}(s) k_{2}(s)-k_{1}(s) k_{2}^{\prime}(s)=0 ;$

(5) $\widetilde{\mathrm{h}}_{v}(\mathrm{~s})=\widetilde{\mathrm{h}}_{v}^{\prime}(\mathrm{s})=\widetilde{\mathrm{h}}_{v}^{\prime \prime}(\mathrm{s})=\widetilde{\mathrm{h}}_{v}^{(3)}(\mathrm{s})=\widetilde{\mathrm{h}}_{v}^{(4)}(\mathrm{s})=0$ if and only if

$$
\begin{array}{r}
\mathbf{v}= \pm \frac{\left|k_{1}(s)\right|}{\sqrt{\left|k_{1}^{2}(s)-k_{2}^{2}(s)\right|}}\left(\frac{k_{2}(s)}{k_{1}(s)} \mathbf{N}_{1}(s)+\mathbf{N}_{2}(s)\right), \\
u=\langle\gamma(s), \mathbf{v}\rangle \text {, and } k_{1}^{\prime}(s) k_{2}(s)-k_{1}(s) k_{2}^{\prime}(s)=\left(k_{1}^{\prime}(s) k_{2}(s)-k_{1}(s) k_{2}^{\prime}(s)\right)^{\prime}=0 .
\end{array}
$$

(D) Suppose that $k_{1}(s) \neq 0$. Then, we have the following claims.

(1) $h_{1 v}(s)=h_{1 v}^{\prime}(s)=0$ if and only if $\mathbf{v}=\mp \mathbf{N}_{2}(s)$;

(2) $h_{1 v}(\mathrm{~s})=\mathrm{h}_{1 v}^{\prime}(\mathrm{s})=\mathrm{h}_{1 v}^{\prime \prime}(\mathrm{s})=0$ if and only if $\mathbf{v}=\mp \mathbf{N}_{2}(\mathrm{~s})$ and $\mathrm{k}_{2}(\mathrm{~s})=0$;

(3) $h_{1 v}(s)=h_{1 v}^{\prime}(s)=h_{1 v}^{\prime \prime}(s)=h_{1 v}^{(3)}(s)=0$ if and only if $\mathbf{v}=\mp \mathbf{N}_{2}(s)$ and $k_{2}(s)=k_{2}^{\prime}(s)=0$.

(E) Suppose that $k_{2}(s) \neq 0$. Then, one has the following claims.

(1) $h_{2 v}(s)=h_{2 v}^{\prime}(s)=0$ if and only if $\mathbf{v}=\mp \mathbf{N}_{1}(s)$;

(2) $h_{2 v}(s)=h_{2 v}^{\prime}(s)=h_{2 v}^{\prime \prime}(s)=0$ if and only if $\mathbf{v}=\mp \mathbf{N}_{1}(s)$ and $k_{1}(s)=0$;

(3) $h_{2 v}(s)=h_{2 v}^{\prime}(s)=h_{2 v}^{\prime \prime}(s)=h_{2 v}^{(3)}(s)=0$ if and only if $\mathbf{v}=\mp \mathbf{N}_{1}(s)$ and $k_{1}(s)=k_{1}^{\prime}(s)=0$.

Proof.

(A) (1) Since $\frac{1}{2} g_{\nu}^{\prime}(s)=\langle\gamma(s)-\mathbf{v}, \mathbf{T}(s)\rangle=0$, we have that there are real numbers $\lambda$ and $\mu$ such that $\gamma(s)-\mathbf{v}=\lambda \mathbf{N}_{1}(\mathrm{~s})+\mu \mathbf{N}_{2}(\mathrm{~s})$. Thus, $g_{v}(\mathrm{~s})=g_{v}^{\prime}(\mathrm{s})=0$ if and only if $\gamma(\mathrm{s})-\mathbf{v}=\lambda \mathbf{N}_{1}(\mathrm{~s})+\mu \mathbf{N}_{2}(\mathrm{~s})$ and $\left\langle\lambda \mathbf{N}_{1}(s)+\mu \mathbf{N}_{2}(s), \lambda \mathbf{N}_{1}(s)+\mu \mathbf{N}_{2}(s)\right\rangle=0$. This is equivalent to the condition that

$$
\gamma(s)-\mathbf{v}=\lambda\left(\mathbf{N}_{1}(s) \pm \mathbf{N}_{2}(s)\right) \text {. }
$$

(2) Since $\frac{1}{2} g_{v}^{\prime \prime}(s)=1+\left\langle\gamma(s)-\mathbf{v}, k_{1}(s) \mathbf{N}_{1}(s)+k_{2}(s) \mathbf{N}_{2}(s)\right\rangle=0$, we have

$$
\lambda\left(\delta\left(\mathbf{N}_{1}(s)\right) k_{1}(s) \pm \delta\left(\mathbf{N}_{2}(s)\right) k_{2}(s)\right)+1=0 .
$$

Since $k_{1}(s) \pm k_{2}(s) \neq 0$, this means that $\lambda=\frac{-1}{\delta\left(\mathbf{N}_{1}(s)\right) k_{1}(s) \pm \delta\left(\mathbf{N}_{2}(s)\right) k_{2}(s)}$. Thus, $g_{v}(s)=g_{v}^{\prime}(s)=g_{v}^{\prime \prime}(s)=0$ if and only if

$$
\gamma(\mathrm{s})-\mathbf{v}=\frac{-1}{\delta\left(\mathbf{N}_{1}(\mathrm{~s})\right) \mathrm{k}_{1}(\mathrm{~s}) \pm \delta\left(\mathbf{N}_{2}(\mathrm{~s})\right) \mathrm{k}_{2}(\mathrm{~s})}\left(\mathbf{N}_{1}(\mathrm{~s}) \pm \mathbf{N}_{2}(\mathrm{~s})\right)
$$

(3) Since

$$
\frac{1}{2} g_{v}^{(3)}(s)=\left\langle\gamma(s)-\mathbf{v}, k_{1}^{\prime}(s) \mathbf{N}_{1}(s)+k_{2}^{\prime}(s) \mathbf{N}_{2}(s)-\left(\delta\left(\mathbf{N}_{1}(s)\right) k_{1}^{2}(s)+\left(\delta\left(\mathbf{N}_{2}(s)\right) k_{2}^{2}(s)\right) \mathbf{T}(s)\right\rangle=0,\right.
$$

we have

$$
\frac{-1}{\delta\left(\mathbf{N}_{1}(s)\right) k_{1}(s) \pm \delta\left(\mathbf{N}_{2}(s)\right) k_{2}(s)} \delta\left(\mathbf{N}_{1}(s)\right) k_{1}^{\prime}(s) \pm\left(\delta\left(\mathbf{N}_{2}(s)\right) k_{2}^{\prime}(s)\right)=0
$$

This means that $\delta\left(\mathbf{N}_{1}(s)\right) k_{1}^{\prime}(s) \pm \delta\left(\mathbf{N}_{2}(s)\right) k_{2}^{\prime}(s)=0$. Thus, $g_{v}(s)=g_{v}^{\prime}(s)=g_{v}^{\prime \prime}(s)=g_{v}^{\prime \prime \prime}(s)=0$ if and only if 


$$
\gamma(s)-\mathbf{v}=\frac{-1}{\delta\left(\mathbf{N}_{1}(s)\right) k_{1}(s) \pm \delta\left(\mathbf{N}_{2}(s)\right) k_{2}(s)}\left(\mathbf{N}_{1}(s) \pm \mathbf{N}_{2}(s)\right)
$$

and

$$
\delta\left(\mathbf{N}_{1}(s)\right) k_{1}^{\prime}(s) \pm \delta\left(\mathbf{N}_{2}(s)\right) k_{2}^{\prime}(s)=0
$$

(4) Since

$$
\frac{1}{2} g_{v}^{(4)}(s)=-\left(\delta\left(\mathbf{N}_{1}(s)\right) k_{1}^{2}(s)+\delta\left(\mathbf{N}_{2}(s)\right) k_{2}^{2}(s)\right)+\left\langle\gamma(s)-\mathbf{v}, \alpha_{1} \mathbf{N}_{1}(s)+\alpha_{2} \mathbf{N}_{2}(s)+\alpha_{3} \mathbf{T}(s)\right\rangle=0,
$$

where

$$
\left\{\begin{array}{l}
\alpha_{1}=\left(k_{1}^{\prime \prime}(s)-\left(\delta\left(\mathbf{N}_{1}(s)\right) k_{1}(s) \pm \delta\left(\mathbf{N}_{2}(s)\right) k_{2}(s)\right) k_{1}(s)\right) \\
\alpha_{2}=\left(k_{2}^{\prime \prime}(s)-\left(\delta\left(\mathbf{N}_{1}(s)\right) k_{1}(s) \pm \delta\left(\mathbf{N}_{2}(s)\right) k_{2}(s)\right) k_{2}(s)\right) \\
\alpha_{3}=-3\left(\delta\left(\mathbf{N}_{1}(s)\right) k_{1}(s) k_{1}^{\prime}(s)+\delta\left(\mathbf{N}_{2}(s)\right) k_{2}(s) k_{2}^{\prime}(s)\right)
\end{array}\right.
$$

we have

$$
-\left(\delta\left(\mathbf{N}_{1}(s)\right) k_{1}^{2}(s)+\left(\delta\left(\mathbf{N}_{2}(s)\right) k_{2}^{2}(s)\right)+\frac{-1}{\delta\left(\mathbf{N}_{1}(\mathbf{s})\right) k_{1}(s) \pm \delta\left(\mathbf{N}_{2}(s)\right) k_{2}(s)} \alpha(s)=0 .\right.
$$

Here

$$
\begin{aligned}
\alpha(s)= & \left(\left(k_{1}^{\prime \prime}(s)-\left(\delta\left(\mathbf{N}_{1}(s)\right) k_{1}(s) \pm\left(\delta\left(\mathbf{N}_{2}(s)\right) k_{2}(s)\right)\right) k_{1}(s)\right)\right. \\
& \left. \pm\left(k_{2}^{\prime \prime}(s)-\left(\left(\delta\left(\mathbf{N}_{1}(s)\right) k_{1}(s) \pm\left(\delta\left(\mathbf{N}_{2}(s)\right) k_{2}(s)\right)\right) k_{2}(s)\right)\right)\right)
\end{aligned}
$$

This means that $\left(k_{1}(s) \mp k_{2}(s)\right)^{2}+\left(k_{1}(s) \mp k_{2}(s)\right)\left(k_{2}^{2}(s)-k_{1}^{2}(s)\right)-\delta\left(\mathbf{N}_{1}(s)\right) k_{1}^{\prime \prime}(s) \mp\left(\delta\left(\mathbf{N}_{2}(s)\right) k_{2}^{\prime \prime}(s)=0\right.$. Thus, $g_{v}(s)=g_{v}^{\prime}(s)=g_{v}^{\prime \prime}(s)=g_{v}^{\prime \prime \prime}(s)=g_{v}^{(4)}(s)=0$ if and only if

$$
\gamma(\mathrm{s})-\mathbf{v}=\frac{-1}{\delta\left(\mathbf{N}_{1}(\mathrm{~s})\right) \mathrm{k}_{1}(\mathrm{~s}) \pm \delta\left(\mathbf{N}_{2}(\mathrm{~s})\right) \mathrm{k}_{2}(\mathrm{~s})}\left(\mathbf{N}_{1}(\mathrm{~s}) \pm \mathbf{N}_{2}(\mathrm{~s})\right),
$$

$\delta\left(\mathbf{N}_{1}(s)\right) k_{1}^{\prime}(s) \pm \delta\left(\mathbf{N}_{2}(s)\right) k_{2}^{\prime}(s)=0$, and

$$
\left(k_{1}(s) \mp k_{2}(s)\right)^{2}+\left(k_{1}(s) \mp k_{2}(s)\right)\left(k_{2}^{2}(s)-k_{1}^{2}(s)\right)-\delta\left(\mathbf{N}_{1}(s)\right) k_{1}^{\prime \prime}(s) \mp \delta\left(\mathbf{N}_{2}(s)\right) k_{2}^{\prime \prime}(s)=0 .
$$

(B) (1) Since $h_{v}^{\prime}(s)=\left\langle\gamma^{\prime}(s), \mathbf{v}\right\rangle=\langle\mathbf{T}(s), \mathbf{v}\rangle=0$, we have that there are real numbers $\lambda$ and $\mu$ such that $\mathbf{v}=\lambda \mathbf{N}_{1}(s)+\mu \mathbf{N}_{2}(s)$. By the condition that $\mathbf{v} \in Q_{\epsilon}^{2}$, we get $\delta\left(\mathbf{N}_{1}(s)\right) \lambda^{2}+\delta\left(\mathbf{N}_{2}(s)\right) \mu^{2}= \pm 1$. The converse direction also holds.

(2) Since $h_{v}^{\prime \prime}(s)=\left\langle k_{1}(s) \mathbf{N}_{1}(s)+k_{2}(s) \mathbf{N}_{2}(s), \mathbf{v}\right\rangle=0, \delta\left(\mathbf{N}_{1}(s)\right) k_{1}(s) \lambda+\delta\left(\mathbf{N}_{2}(s)\right) k_{2}(s) \mu=0$. It follows from the fact $\delta\left(\mathbf{N}_{1}(s)\right) \lambda^{2}+\delta\left(\mathbf{N}_{2}(s)\right) \mu^{2}= \pm 1$ that

$$
\mu= \pm \frac{\left|k_{1}(s)\right|}{\sqrt{\left|\delta\left(\mathbf{N}_{2}(s)\right) k_{1}^{2}(s)+\delta\left(\mathbf{N}_{1}(s)\right) k_{2}^{2}(s)\right|}}= \pm \frac{\left|k_{1}(s)\right|}{\sqrt{\left|k_{1}^{2}(s)-k_{2}^{2}(s)\right|}} .
$$

Therefore, we have

$$
\mathbf{v}= \pm \frac{\left|k_{1}(s)\right|}{\sqrt{\left|k_{1}^{2}(s)-k_{2}^{2}(s)\right|}}\left(\frac{k_{2}(s)}{k_{1}(s)} \mathbf{N}_{1}(s)+\mathbf{N}_{2}(s)\right) .
$$

(3) Since $h_{v}^{(3)}(s)=\left\langle k_{1}^{\prime}(s) \mathbf{N}_{1}(s)+k_{2}^{\prime}(s) \mathbf{N}_{2}(s)-\left(\delta\left(\mathbf{N}_{1}(s)\right) k_{1}^{2}(s)+\delta\left(\mathbf{N}_{2}(s)\right) k_{2}^{2}(s)\right) \mathbf{T}, \mathbf{v}\right\rangle=0$, we have

$$
\pm \frac{\left|k_{1}(s)\right|}{\sqrt{\left|k_{1}^{2}(s)-k_{2}^{2}(s)\right|}}\left(\frac{\delta\left(\mathbf{N}_{1}(s)\right) k_{1}^{\prime}(s) k_{2}(s)+\delta\left(\mathbf{N}_{2}(s)\right) k_{1}(s) k_{2}^{\prime}(s)}{k_{1}(s)}\right)=0 .
$$

By the condition of (3), this is equivalent to the condition $k_{1}^{\prime}(s) k_{2}(s)-k_{1}(s) k_{2}^{\prime}(s)=0$. 
(4) Since $h_{v}^{(4)}(s)=\left\langle\beta_{1} \mathbf{N}_{1}(s)+\beta_{2} \mathbf{N}_{2}(s)-\beta_{3} \mathbf{T}(s), \mathbf{v}\right\rangle=0$, where

$$
\left\{\begin{array}{l}
\beta_{1}=\left(k_{1}^{\prime \prime}(s)-\delta\left(\mathbf{N}_{1}(s)\right) k_{1}^{3}(s)-\delta\left(\mathbf{N}_{2}(s)\right) k_{1}(s) k_{2}^{2}(s)\right), \\
\beta_{2}=\left(k_{2}^{\prime \prime}(s)-\delta\left(\mathbf{N}_{1}(s)\right) k_{1}^{2}(s) k_{2}(s)-\delta\left(\mathbf{N}_{2}(s)\right) k_{2}^{3}(s)\right), \\
\beta_{3}=3\left(\delta\left(\mathbf{N}_{1}(s)\right) k_{1}(s) k_{1}^{\prime}(s)+\delta\left(\mathbf{N}_{2}(s)\right) k_{2}(s) k_{2}^{\prime}(s)\right),
\end{array}\right.
$$

we have

$$
\pm \frac{\left|k_{1}(s)\right|}{\sqrt{\left|k_{1}^{2}(s)-k_{2}^{2}(s)\right|}}\left(\frac{\delta\left(\mathbf{N}_{1}(s)\right) k_{1}^{\prime \prime}(s) k_{2}(s)+\delta\left(\mathbf{N}_{2}(s)\right) k_{1}(s) k_{2}^{\prime \prime}(s)}{k_{1}(s)}\right)=0 .
$$

By the condition of (4), this is equivalent to the condition

$$
k_{1}^{\prime}(s) k_{2}(s)-k_{1}(s) k_{2}^{\prime}(s)=\left(k_{1}^{\prime}(s) k_{2}(s)-k_{1}(s) k_{2}^{\prime}(s)\right)^{\prime}=0 .
$$

(C) The proof of (C) follows from the proof of (B), so we omit it.

(D) (1) If

$$
\mathrm{h}_{1 v}(\mathrm{~s})=\left\langle\mathbf{N}_{1}(\mathrm{~s}), \mathbf{v}\right\rangle=0,
$$

then we have that there are real numbers $\lambda$ and $\mu$ such that $\mathbf{v}=\lambda \mathbf{T}(\mathrm{s})+\mu \mathbf{N}_{2}(\mathrm{~s})$. Moreover, in combination with $\mathbf{v} \in Q_{\epsilon}^{2}, \lambda^{2}+\delta\left(\mathbf{N}_{2}(s)\right) \mu^{2}= \pm 1$. It follows that $h_{1 v}(s)=0$ if and only if $\mathbf{v}=\lambda \mathbf{T}(s)+\mu \mathbf{N}_{2}(s)$ and $\lambda^{2}+\delta\left(\mathbf{N}_{2}(s)\right) \mu^{2}= \pm 1$. When $h_{1 v}(s)=0$, the assertion (1) follows from the fact that

$$
h_{1 v}^{\prime}(s)=\left\langle-\delta\left(\mathbf{N}_{1}(s)\right) k_{1}(s) \mathbf{T}(s), \mathbf{v}\right\rangle=-\delta\left(\mathbf{N}_{1}(s)\right) k_{1}(s) \lambda
$$

and $k_{1}(s) \neq 0$. Thus, we get that $h_{1 v}(s)=h_{1 v}^{\prime}(s)=0$ if and only if $\mathbf{v}=\mp \mathbf{N}_{2}(s)$.

(2) When $h_{1 v}(s)=h_{1 v}^{\prime}(s)=0$, the assertion (2) follows from the fact that

$$
h_{1 v}^{\prime \prime}(s)=\left\langle-\delta\left(\mathbf{N}_{1}(s)\right) k_{1}^{\prime}(s) T(s)-\delta\left(\mathbf{N}_{1}(s)\right) k_{1}^{2}(s) \mathbf{N}_{1}(s)-\delta\left(\mathbf{N}_{1}(s)\right) k_{1}(s) k_{2}(s) \mathbf{N}_{2}(s), \mp \mathbf{N}_{2}(s)\right\rangle=\mp k_{1}(s) k_{2}(s)
$$

and $k_{1}(s) \neq 0$.

(3) Under the condition that $h_{1 v}(s)=h_{1 v}^{\prime}(s)=h_{1 v}^{\prime \prime}(s)=0$, this derivative is computed as follows: $h_{1 v}^{(3)}(s)=\left\langle\left(-\delta\left(\mathbf{N}_{1}(s)\right) k_{1}^{\prime \prime}(s)+k_{1}^{3}(s)-k_{1}(s) k_{2}^{2}(s)\right) \mathbf{T}(s)-3 \delta\left(\mathbf{N}_{1}(s)\right) k_{1}(s) k_{1}^{\prime}(s) \mathbf{N}_{1}(s)-\delta\left(\mathbf{N}_{1}(s)\right)\left(2 k_{1}^{\prime}(s) k_{2}(s)+\right.\right.$ $\left.\left.k_{1}(s) k_{2}^{\prime}(s)\right) \mathbf{N}_{2}(s), \mp \mathbf{N}_{2}(s)\right\rangle=\mp\left(2 k_{1}^{\prime}(s) k_{2}(s)+k_{1}(s) k_{2}^{\prime}(s)\right)=\mp k_{1}(s) k_{2}^{\prime}(s)$. Since $k_{1}(s) \neq 0$, we get that $h_{1 v}^{(3)}(s)=0$ is equivalent to the condition $k_{2}^{\prime}(s)=0$. The assertion (3) follows.

(E) Using the same computation as the proof of (D), we can get (E).

Proposition 3.3. Let $\gamma: \mathrm{I} \rightarrow \mathbb{R}_{1}^{3}$ be a unit speed regular spacelike curve, then we have the following results.

(1) Suppose $\delta\left(\mathbf{N}_{1}(s)\right) k_{1}(s) \pm \delta\left(\mathbf{N}_{2}(s)\right) k_{2}(s) \neq 0$. Then $\delta\left(\mathbf{N}_{1}(s)\right) k_{1}^{\prime}(s) \pm \delta\left(\mathbf{N}_{2}(s)\right) k_{2}^{\prime}(s)=0$ if and only if each of

$$
p_{ \pm}=\gamma(s)+\frac{1}{\delta\left(\mathbf{N}_{1}(s)\right) k_{1}(s) \pm \delta\left(\mathbf{N}_{2}(s)\right) k_{2}(s)}\left(\mathbf{N}_{1}(s) \pm \mathbf{N}_{2}(s)\right)
$$

is a constant vector.

(2) Suppose $k_{1}(s) \neq 0$. Then $k_{1}(s) k_{2}^{\prime}(s)-k_{1}^{\prime}(s) k_{2}(s)=0$ if and only if each of

$$
\mathbf{v}=\mp \frac{\left|k_{1}(s)\right|}{\sqrt{\left|k_{1}^{2}(s)-k_{2}^{2}(s)\right|}}\left(\frac{k_{2}(s)}{k_{1}(s)} \mathbf{N}_{1}(s)+\mathbf{N}_{2}(s)\right)
$$

is a constant vector.

(3) Suppose $k_{1}(s) \neq 0$. Then, $k_{2}(s)=0$ if and only if each of $\mathbf{v}=\mp \mathbf{N}_{2}(s)$ is a constant vector.

(4) Suppose $k_{2}(s) \neq 0$. Then, $k_{1}(s)=0$ if and only if each of $\mathbf{v}=\mp \mathbf{N}_{1}(s)$ is a constant vector. 
Then, we have the following proposition.

Proposition 3.4. We consider functions defined in Proposition 3.2. Then we have the following claims.

(1) If $g_{v_{0}}(s)$ has $A_{k}$-singularity $(k=1,2,3)$ at $s_{0}$, then $G(s, v)$ is an $\mathcal{R}$-versal unfoldings of $g_{v_{0}}(s)$.

(2) If $h_{v_{0}}(s)$ has $A_{k}$-singularity $(k=2,3)$ at $s_{0}$, then $\mathrm{H}(s, \mathbf{v})$ is an $\mathcal{R}^{+}$-versal unfolding of $\mathrm{h}_{\mathrm{v}_{0}}(\mathrm{~s})$.

(3) If $\widetilde{\mathrm{h}}_{\mathrm{v}_{\mathbf{0}}}(\mathrm{s})$ has $A_{\mathrm{k}}$-singularity $(\mathrm{k}=2,3)$ at $\mathrm{s}_{0}$, then $\widetilde{\mathrm{H}}(\mathrm{s}, \mathbf{v})$ is an $\mathcal{R}$-versal unfolding of $\widetilde{\mathrm{h}}_{\mathrm{v}_{\mathbf{0}}}(\mathrm{s})$.

(4) If $\mathrm{h}_{1 v_{0}}(\mathrm{~s})$ has $\mathrm{A}_{\mathrm{k}}$-singularity $(\mathrm{k}=1,2)$ at $\mathrm{s}_{0}$, then $\mathrm{H}_{1}(\mathrm{~s}, \mathbf{v})$ is an $\mathcal{R}$-versal unfolding of $\mathrm{h}_{1 v_{0}}(\mathrm{~s})$.

(5) If $h_{2 v_{0}}(\mathrm{~s})$ has $A_{k}$-singularity $(k=1,2)$ at $s_{0}$, then $\mathrm{H}_{2}(\mathrm{~s}, \mathbf{v})$ is an $\mathcal{R}$-versal unfolding of $\mathrm{h}_{2 v_{0}}(\mathrm{~s})$.

Proof.

(1) We denote that $\gamma(s)=\left(x_{1}(s), x_{2}(s), x_{3}(s)\right)$ and $\mathbf{v}=\left(v_{1}, v_{2}, v_{3}\right)$. Under this notation we have

$$
\mathrm{G}(\mathrm{s}, \mathbf{v})=-\left(\mathrm{x}_{1}(\mathrm{~s})-v_{1}\right)^{2}+\left(\mathrm{x}_{2}(\mathrm{~s})-v_{2}\right)^{2}+\left(\mathrm{x}_{3}(\mathrm{~s})-v_{3}\right)^{2} \text {. }
$$

Thus we have

$$
\begin{aligned}
\frac{\partial G}{\partial v_{1}}(s, \mathbf{v}) & =2\left(x_{1}(s)-v_{1}\right), & \frac{\partial G}{\partial v_{i}}(s, \mathbf{v}) & =-2\left(x_{i}(s)-v_{i}\right), i=2,3, \\
\frac{\partial}{\partial s} \frac{\partial G}{\partial v_{1}}(s, \mathbf{v}) & =2 x_{1}^{\prime}(s), & \frac{\partial}{\partial s} \frac{\partial G}{\partial v_{i}}(s, \mathbf{v}) & =-2 x_{i}^{\prime}(s), i=2,3, \\
\frac{\partial^{2}}{\partial s^{2}} \frac{\partial G}{\partial v_{1}}(s, \mathbf{v}) & =2 x_{1}^{\prime \prime}(s), & \frac{\partial^{2}}{\partial s^{2}} \frac{\partial G}{\partial v_{i}}(s, \mathbf{v}) & =-2 x_{i}^{\prime \prime}(s), i=2,3 .
\end{aligned}
$$

Therefore the 2-jet of $\frac{\partial G}{\partial v_{i}}(s, \mathbf{v})(i=1,2,3)$ at $s_{0}$ is given by

$$
j^{2}\left(\frac{\partial G}{\partial v_{i}}\left(s, \mathbf{v}_{0}\right)\right)\left(s_{0}\right)=\frac{\partial}{\partial s} \frac{\partial G}{\partial v_{i}}\left(s-s_{0}\right)+\frac{1}{2} \frac{\partial^{2}}{\partial s^{2}} \frac{\partial G}{\partial v_{i}}\left(s-s_{0}\right)^{2}=a_{1 i}\left(s-s_{0}\right)+\frac{1}{2} a_{2 i}\left(s-s_{0}\right)^{2} .
$$

It is enough to show that the rank of the matrix $A$ is 3 , where

$$
A=\left(\begin{array}{ccc}
2\left(x_{1}\left(s_{0}\right)-v_{1}\right) & -2\left(x_{2}\left(s_{0}\right)-v_{2}\right) & -2\left(x_{3}\left(s_{0}\right)-v_{3}\right) \\
2 x_{1}^{\prime}\left(s_{0}\right) & -2 x_{2}^{\prime}\left(s_{0}\right) & -2 x_{3}^{\prime}\left(s_{0}\right) \\
2 x_{1}^{\prime \prime}\left(s_{0}\right) & -2 x_{2}^{\prime \prime}\left(s_{0}\right) & -2 x_{3}^{\prime \prime}\left(s_{0}\right)
\end{array}\right)
$$

Then we have

$$
\begin{aligned}
\operatorname{det} A & =8 \operatorname{det}\left(\boldsymbol{\gamma}\left(\mathrm{s}_{0}\right)-\mathbf{v}, \mathbf{T}\left(\mathrm{s}_{0}\right), \mathbf{T}^{\prime}\left(\mathrm{s}_{0}\right)\right) \\
& =8 \operatorname{det}\left(\frac{-1}{\delta\left(\mathbf{N}_{1}\left(\mathrm{~s}_{0}\right)\right) \mathrm{k}_{1}\left(\mathrm{~s}_{0}\right) \pm \delta\left(\mathbf{N}_{2}\left(\mathrm{~s}_{0}\right)\right) \mathrm{k}_{2}\left(\mathrm{~s}_{0}\right)}\left(\mathbf{N}_{1}\left(\mathrm{~s}_{0}\right) \pm \mathbf{N}_{2}\left(\mathrm{~s}_{0}\right)\right), \mathbf{T}, \mathrm{k}_{1}\left(\mathrm{~s}_{0}\right) \mathbf{N}_{1}+\mathrm{k}_{2}\left(\mathrm{~s}_{0}\right) \mathbf{N}_{2}\right) \\
& =\frac{8}{\delta\left(\mathbf{N}_{1}\left(\mathrm{~s}_{0}\right)\right) \mathrm{k}_{1}\left(\mathrm{~s}_{0}\right) \pm \delta\left(\mathbf{N}_{2}\left(\mathrm{~s}_{0}\right)\right) \mathrm{k}_{2}\left(\mathrm{~s}_{0}\right)}\left(\mathrm{k}_{2}\left(\mathrm{~s}_{0}\right) \mp \mathrm{k}_{1}\left(\mathrm{~s}_{0}\right)\right) \\
& = \pm 8 \neq 0,
\end{aligned}
$$

which implies that the rank of $A$ is 3 . If we consider the matrix which consists of the first and the second rows of the matrix $A$, so that the rank of this matrix is two. This completes the proof.

(2) We denote that $\gamma(s)=\left(x_{1}(s), x_{2}(s), x_{3}(s)\right)$, and $\mathbf{v}=\left(v_{1}, v_{2}, \pm \sqrt{ \pm 1+v_{1}^{2}-v_{2}^{2}}\right)$. Under this notation we have

$$
\mathrm{H}(\mathrm{s}, \mathbf{v})=-\mathrm{x}_{1}(\mathrm{~s}) v_{1}+\mathrm{x}_{2}(\mathrm{~s}) v_{2} \pm \mathrm{x}_{3}(\mathrm{~s}) \sqrt{ \pm 1+v_{1}^{2}-v_{2}^{2}}
$$

Thus we have

$$
\frac{\partial \mathrm{H}}{\partial v_{1}}=-\mathrm{x}_{1}(\mathrm{~s}) \pm \frac{v_{1} \mathrm{x}_{3}(\mathrm{~s})}{\sqrt{ \pm 1+v_{1}^{2}-v_{2}^{2}}}, \quad \frac{\partial \mathrm{H}}{\partial v_{2}}=\mathrm{x}_{2}(\mathrm{~s}) \mp \frac{v_{2} \mathrm{x}_{3}(\mathrm{~s})}{\sqrt{ \pm 1+v_{1}^{2}-v_{2}^{2}}}
$$




$$
\begin{array}{rlrl}
\frac{\partial}{\partial s} \frac{\partial H}{\partial v_{1}} & =-x_{1}^{\prime}(\mathrm{s}) \pm \frac{v_{1} x_{3}^{\prime}(s)}{\sqrt{ \pm 1+v_{1}^{2}-v_{2}^{2}}}, & \frac{\partial}{\partial s} \frac{\partial \mathrm{H}}{\partial v_{2}}=x_{2}^{\prime}(\mathrm{s}) \mp \frac{v_{2} x_{3}^{\prime}(\mathrm{s})}{\sqrt{ \pm 1+v_{1}^{2}-v_{2}^{2}}}, \\
\frac{\partial^{2}}{\partial s^{2}} \frac{\partial H}{\partial v_{1}}=-x_{1}^{\prime \prime}(\mathrm{s}) \pm \frac{v_{1} x_{3}^{\prime \prime}(s)}{\sqrt{ \pm 1+v_{1}^{2}-v_{2}^{2}}}, & \frac{\partial^{2}}{\partial s^{2}} \frac{\partial H}{\partial v_{2}}=x_{2}^{\prime \prime}(s) \mp \frac{v_{2} x_{3}^{\prime \prime}(s)}{\sqrt{ \pm 1+v_{1}^{2}-v_{2}^{2}}} .
\end{array}
$$

Therefore the 2-jet of $\frac{\partial H}{\partial v_{i}}(s, v)(i=1,2)$ at $s_{0}$ is given by

$$
j^{2}\left(\frac{\partial H}{\partial v_{i}}\left(s, v_{0}\right)\right)\left(s_{0}\right)=\frac{\partial}{\partial s} \frac{\partial H}{\partial v_{i}}\left(s-s_{0}\right)+\frac{1}{2} \frac{\partial^{2}}{\partial s^{2}} \frac{\partial H}{\partial v_{i}}\left(s-s_{0}\right)^{2}=a_{1 i}\left(s-s_{0}\right)+\frac{1}{2} a_{2 i}\left(s-s_{0}\right)^{2} .
$$

It is enough to show that the rank of the matrix $B$ is 2 , where

$$
B=\left(\begin{array}{cc}
-x_{1}^{\prime}\left(s_{0}\right) \pm \frac{v_{1} x_{3}^{\prime}\left(s_{0}\right)}{\sqrt{ \pm 1+v_{1}^{2}-v_{2}^{2}}} & x_{2}^{\prime}\left(s_{0}\right) \mp \frac{v_{2} x_{3}^{\prime}\left(s_{0}\right)}{\sqrt{ \pm 1+v_{1}^{2}-v_{2}^{2}}} \\
-x_{1}^{\prime \prime}\left(s_{0}\right) \pm \frac{v_{1} x_{3}^{\prime \prime}\left(s_{0}\right)}{\sqrt{ \pm 1+v_{1}^{2}-v_{2}^{2}}} & x_{2}^{\prime \prime}\left(s_{0}\right) \mp \frac{v_{2} x_{3}^{\prime \prime}\left(s_{0}\right)}{\sqrt{ \pm 1+v_{1}^{2}-v_{2}^{2}}}
\end{array}\right) .
$$

We have

$$
\begin{aligned}
\operatorname{det} B & =\frac{v_{1}}{v_{3}}\left(x_{2}^{\prime \prime} x_{3}^{\prime}-x_{2}^{\prime} x_{3}^{\prime \prime}\right)\left(s_{0}\right)+\frac{v_{2}}{v_{3}}\left(x_{1}^{\prime} x_{3}^{\prime \prime}-x_{1}^{\prime \prime} x_{3}^{\prime}\right)\left(s_{0}\right)-\left(x_{1}^{\prime} x_{2}^{\prime \prime}-x_{1}^{\prime \prime} x_{2}^{\prime}\right)\left(s_{0}\right) \\
& =-\frac{1}{v_{3}}\left\langle\mathbf{v}, \mathbf{T}\left(s_{0}\right) \wedge \mathbf{T}^{\prime}\left(s_{0}\right)\right\rangle \\
& =-\frac{1}{v_{3}}\left\langle\mathbf{v}, \mathbf{T}\left(s_{0}\right) \wedge k_{1}\left(s_{0}\right) \mathbf{N}_{1}\left(s_{0}\right)+k_{2}\left(s_{0}\right) \mathbf{N}_{2}\left(s_{0}\right)\right\rangle \\
& =-\frac{1}{v_{3}}\left\langle\mathbf{v}, k_{1}\left(s_{0}\right) \delta\left(\mathbf{N}_{2}\left(s_{0}\right)\right) \mathbf{N}_{2}\left(s_{0}\right)-k_{2}\left(s_{0}\right) \delta\left(\mathbf{N}_{1}\left(s_{0}\right)\right) \mathbf{N}_{1}\left(s_{0}\right)\right\rangle \\
& =-\frac{1}{v_{3}}\left\langle \pm \frac{\left|k_{1}\left(s_{0}\right)\right|}{\sqrt{\left|k_{1}^{2}\left(s_{0}\right)-k_{2}^{2}\left(s_{0}\right)\right|}}\left(\frac{k_{2}\left(s_{0}\right)}{k_{1}\left(s_{0}\right)} \mathbf{N}_{1}+\mathbf{N}_{2}\right), k_{1}\left(s_{0}\right) \delta\left(\mathbf{N}_{2}\right) \mathbf{N}_{2}-k_{2}\left(s_{0}\right) \delta\left(\mathbf{N}_{1}\right) \mathbf{N}_{1}\right\rangle \\
& =\mp \operatorname{sgn}\left(k_{1}\left(s_{0}\right)\right) \frac{1}{v_{3}} \frac{k_{1}^{2}\left(s_{0}\right)-k_{2}^{2}\left(s_{0}\right)}{\sqrt{\left|k_{1}^{2}\left(s_{0}\right)-k_{2}^{2}\left(s_{0}\right)\right|}} \neq 0 .
\end{aligned}
$$

Note that $\mathbf{v}_{0} \in \mathfrak{B}_{\mathrm{H}}$ is a singular point, where

$$
\mathbf{v}= \pm \frac{\left|k_{1}\left(s_{0}\right)\right|}{\sqrt{\left|k_{1}^{2}\left(s_{0}\right)-k_{2}^{2}\left(s_{0}\right)\right|}}\left(\frac{k_{2}\left(s_{0}\right)}{k_{1}\left(s_{0}\right)} \mathbf{N}_{1}\left(s_{0}\right)+\mathbf{N}_{2}\left(s_{0}\right)\right) .
$$

This completes the proof.

(3) Under the same notations as (2), we have

$$
\widetilde{\mathrm{H}}(s, \mathbf{v})=-\chi_{1}(s) v_{1}+\chi_{2}(s) v_{2} \pm x_{3}(s) \sqrt{ \pm 1+v_{1}^{2}-v_{2}^{2}}-u .
$$

It is enough to show that the rank of the matrix $C$ is 2 , where

$$
C=\left(\begin{array}{ccc}
-x_{1}^{\prime}(s) \pm \frac{v_{1} x_{3}^{\prime}(s)}{\sqrt{ \pm 1+v_{1}^{2}-v_{2}^{2}}} & x_{2}^{\prime}(s) \mp \frac{v_{2} x_{3}^{\prime}(s)}{\sqrt{ \pm 1+v_{1}^{2}-v_{2}^{2}}} & -1 \\
-\chi_{1}^{\prime \prime}(s) \pm \frac{v_{1} x_{3}^{\prime \prime}(s)}{\sqrt{ \pm 1+v_{1}^{2}-v_{2}^{2}}} & \chi_{2}^{\prime \prime}(s) \mp \frac{v_{2} x_{3}^{\prime \prime}(s)}{\sqrt{ \pm 1+v_{1}^{2}-v_{2}^{2}}} & 0
\end{array}\right)
$$

This follows from the proof of (2). Thus, we complete the proof of (3). 
(4) We denote that $\mathbf{N}_{1}(s)=\left(\mathrm{n}_{11}(\mathrm{~s}), \mathrm{n}_{12}(\mathrm{~s}), \mathrm{n}_{13}(\mathrm{~s})\right), \mathbf{v}=\left(v_{1}, v_{2}, \pm \sqrt{ \pm 1+v_{1}^{2}-v_{2}^{2}}\right)$. Under this notation, we have that

$$
\mathrm{H}_{1}(\mathrm{~s}, \mathbf{v})=-\mathrm{n}_{11}(\mathrm{~s}) v_{1}+\mathrm{n}_{12}(\mathrm{~s}) v_{2} \pm \mathrm{n}_{13}(\mathrm{~s}) \sqrt{ \pm 1+v_{1}^{2}-v_{2}^{2}}
$$

Thus, we have that

$$
\begin{aligned}
\frac{\partial \mathrm{H}_{1}}{\partial v_{1}} & =-\mathrm{n}_{11}(\mathrm{~s}) \pm \frac{v_{\mathrm{i}} \mathrm{n}_{13}(\mathrm{~s})}{\sqrt{ \pm 1+v_{1}^{2}-v_{2}^{2}}}, & \frac{\partial \mathrm{H}_{1}}{\partial v_{2}} & =n_{12}(\mathrm{~s}) \mp \frac{v_{i} \mathrm{n}_{13}(\mathrm{~s})}{\sqrt{ \pm 1+v_{1}^{2}-v_{2}^{2}}}, \\
\frac{\partial}{\partial s} \frac{\partial \mathrm{H}_{1}}{\partial v_{1}} & =-\mathrm{n}_{11}^{\prime}(\mathrm{s}) \pm \frac{v_{\mathrm{i}} \mathrm{n}_{13}^{\prime}(\mathrm{s})}{\sqrt{ \pm 1+v_{1}^{2}-v_{2}^{2}}}, & \frac{\partial}{\partial s} \frac{\partial \mathrm{H}_{1}}{\partial v_{2}} & =n_{12}^{\prime}(\mathrm{s}) \mp \frac{v_{i} \mathrm{n}_{13}^{\prime}(\mathrm{s})}{\sqrt{ \pm 1+v_{1}^{2}-v_{2}^{2}}} .
\end{aligned}
$$

It is enough to show that the rank of the matrix $D$ is 2 , where

$$
\mathrm{D}=\left(\begin{array}{cc}
-\mathrm{n}_{11}\left(s_{0}\right) \pm \frac{v_{1} n_{13}\left(s_{0}\right)}{\sqrt{ \pm 1+v_{1}^{2}-v_{2}^{2}}} & n_{12}\left(s_{0}\right) \mp \frac{v_{2} n_{13}\left(s_{0}\right)}{\sqrt{ \pm 1+v_{1}^{2}-v_{2}^{2}}} \\
-n_{11}^{\prime}\left(s_{0}\right) \pm \frac{v_{1} n_{13}^{\prime}\left(s_{0}\right)}{\sqrt{ \pm 1+v_{1}^{2}-v_{2}^{2}}} & n_{12}^{\prime}\left(s_{0}\right) \mp \frac{v_{2} n_{13}^{\prime}\left(s_{0}\right)}{\sqrt{ \pm 1+v_{1}^{2}-v_{2}^{2}}}
\end{array}\right) .
$$

Denote that $v_{3}= \pm \sqrt{ \pm 1+v_{1}^{2}-v_{2}^{2}}$. Then, we have that

$$
\begin{aligned}
\operatorname{det} D & =-\left(n_{12}^{\prime} n_{11}-n_{12} n_{11}^{\prime}\right)\left(s_{0}\right)+\frac{v_{1}}{v_{3}}\left(n_{12}^{\prime} n_{13}-n_{12} n_{13}^{\prime}\right)\left(s_{0}\right)+\frac{v_{2}}{v_{3}}\left(n_{13}^{\prime} n_{11}-n_{13} n_{11}^{\prime}\right)\left(s_{0}\right) \\
& =-\frac{1}{v_{3}}\left\langle\mathbf{v}, \mathbf{N}_{1}\left(s_{0}\right) \wedge \mathbf{N}_{1}^{\prime}\left(s_{0}\right)\right\rangle \\
& =-\frac{1}{v_{3}}\left\langle\mathbf{v}, \mathbf{N}_{1}\left(s_{0}\right) \wedge-\delta\left(\mathbf{N}_{1}\left(s_{0}\right)\right) k_{1}\left(s_{0}\right) \mathbf{T}\left(s_{0}\right)\right\rangle \\
& =-\frac{1}{v_{3}}\left\langle\mathbf{v},-k_{1}\left(s_{0}\right) \mathbf{N}_{2}\left(s_{0}\right)\right\rangle \\
& =-\frac{1}{v_{3}}\left\langle\mp \mathbf{N}_{2}\left(s_{0}\right),-k_{1}\left(s_{0}\right) \mathbf{N}_{2}\left(s_{0}\right)\right\rangle=\mp \delta\left(\mathbf{N}_{2}\left(s_{0}\right)\right) \frac{1}{v_{3}} k_{1}\left(s_{0}\right) \neq 0 .
\end{aligned}
$$

Note that $\mathbf{v} \in \mathfrak{D}_{\mathrm{H}_{1}}$ is a singular point, where

$$
\mathbf{v}=\mp \mathbf{N}_{2}\left(s_{0}\right) .
$$

This completes the proof.

(5) Using the same computation as the proof of (4), we can get (5).

\section{Proof of the main results}

Proposition 3.4 means that those functions are generating families and now we can apply the above arguments to our situation. By Proposition 3.2 and the definitions of wave fronts and caustics, we can prove Proposition 2.2.

Proof of Proposition 2.2. Taking $G(s, \mathbf{v})=-\left(x_{1}(s)-v_{1}\right)^{2}+\left(x_{2}(s)-v_{2}\right)^{2}+\left(x_{3}(s)-v_{3}\right)^{2}$ in Proposition 3.4 for example, we get the Jacobi matrix of $\Delta^{*} G=\left(G, \frac{\partial G}{\partial s}\right)$ as

$$
\mathrm{J} \Delta^{*} \mathrm{G}=\left(\begin{array}{cccc}
2\left\langle\gamma\left(s_{0}\right)-\mathbf{v}, \mathbf{t}\left(s_{0}\right)\right\rangle & 2\left(x_{1}\left(s_{0}\right)-v_{1}\right) & -2\left(x_{2}\left(s_{0}\right)-v_{2}\right) & -2\left(x_{3}\left(s_{0}\right)-v_{3}\right) \\
2+2\left\langle\gamma\left(s_{0}\right)-\mathbf{v}, \mathbf{t}^{\prime}\left(s_{0}\right)\right\rangle & 2 x_{1}^{\prime}\left(s_{0}\right) & -2 x_{2}^{\prime}\left(s_{0}\right) & -2 x_{3}^{\prime}\left(s_{0}\right)
\end{array}\right) .
$$

Since the rank of the matrix $A$ in the proof of Proposition $3.4(1)$ is 3 , the rank of the matrix $J \Delta^{*} G=2$. 
This means that $G(s, \mathbf{v})$ is a Morse family. In this case we have a smooth 2-dimensional submanifold,

$$
\begin{aligned}
\Sigma_{*}(G) & =\left\{(s, \mathbf{v}) \in\left(\mathbb{R} \times \mathbb{R}^{3}, \mathbf{0}\right) \mid G(s, \mathbf{v})=\frac{\partial G}{\partial s}(s, \mathbf{v})=0\right\} \\
& =\left\{(s, \mathbf{v}) \in\left(\mathbb{R} \times \mathbb{R}^{3}, \mathbf{0}\right) \mid \mathbf{v}=\boldsymbol{\gamma}(s)+u\left(\mathbf{N}_{1}(s) \pm \mathbf{N}_{2}(s)\right), s \in I\right\} .
\end{aligned}
$$

We denote $\pi: \mathrm{PT}^{*} \mathbb{R}_{1}^{3} \rightarrow \mathbb{R}_{1}^{3}$ by the canonical projection, then the map germ $\Phi_{\mathrm{G}}:\left(\Sigma_{*}(\mathrm{G}), \mathbf{0}\right) \longrightarrow \mathrm{PT}^{*} \mathbb{R}_{1}^{3}$ defined by

$$
\Phi_{\mathrm{G}}(\mathrm{s}, \mathbf{v})=\left(\mathbf{v},\left[\left(\mathrm{x}_{1}(\mathrm{~s})-v_{1}\right):-\left(\mathrm{x}_{2}(\mathrm{~s})-v_{2}\right):-\left(\mathrm{x}_{3}(\mathrm{~s})-v_{3}\right)\right]\right)
$$

is a Legendrian immersion germ and $G$ is a generating family of $\Phi_{G}\left(\Sigma_{*} G\right)$. So the map

$$
\pi \circ \Phi_{\mathrm{G}}(\mathrm{s}, \mathbf{v}): \Sigma_{*}(\mathrm{G}) \rightarrow \mathbb{R}_{1}^{3}
$$

given by $(s, \mathbf{v}) \rightarrow \mathbf{v}$ is a Legendrian map. The wave front

$$
\mathrm{W}\left(\Phi_{\mathrm{G}}\right)=\left\{\mathbf{v} \in \mathbb{R}^{3} \mid \mathbf{v}=\boldsymbol{\gamma}(\mathrm{s})+\mathfrak{u}\left(\mathbf{N}_{1}(\mathrm{~s}) \pm \mathbf{N}_{2}(\mathrm{~s})\right), \mathrm{s} \in \mathrm{I}\right\}
$$

of $\gamma(s)$ is the set of critical values of the Legendrian map $\pi \circ \Phi_{G}(s, \mathbf{v})$, and is precisely $D_{G}$. As you can see that the parametrisations of the wave fronts are just the parallel developables given by

$$
\mathcal{P} \mathcal{L} \mathcal{D}_{\gamma}^{ \pm}(s, u)=\gamma(s)+u\left(\mathbf{N}_{1}(s) \pm \mathbf{N}_{2}(s)\right)
$$

It follows that for a generic curve, the wave front $\mathrm{W}\left(\Phi_{\mathrm{G}}\right)$ of $\gamma(\mathrm{s})$ is locally either a regular surface, or has cuspidaledge singularity, or swallowtail. The local models of the wave front at $\mathbf{v}$ corresponding to $s \in \mathrm{I}$ depend on the $\mathcal{R}$-singularity type of $g_{v}(s)$ at $s$. For a generic $\gamma(s), g_{v}(s)$ has local singularities of types $A_{1}, A_{2}$, or $A_{3}$. The wave front is a regular surface at an $A_{1}$-singularity of $g_{v}(s)$. It is a cupidaledge at an $A_{2}$-singularity of $g_{v}(s)$ and has swallowtail singularity at an $A_{3}$-singularity of $g_{v}(s)$. If we apply the same arguments as $G(s, \mathbf{v})$ to the functions $\widetilde{H}, H_{1}$, and $H_{2}$, respectively, we can get that dual surfaces are two dimensional wave fronts which have $A_{1}$-singularity, $A_{2}$-singularity, and $A_{3}$-singularity. Bishop pseudospherical images are one dimensional wave fronts which have $A_{1}$-singularity and $A_{2}$-singularity. Bishop pseudo-spherical Darboux images can be seen as one dimensional caustics which have $A_{2}$-singularity and $A_{3}$-singularity in the framework of the theory of Lagrangian singularity.

Proof of Theorem 2.3.

(1) First, we consider the assertion (A). Let $\gamma: I \rightarrow \mathbb{R}_{1}^{3}$ be a regular unit speed spacelike curve with $\delta\left(\mathbf{N}_{1}(s)\right) k_{1}(s) \pm \delta\left(\mathbf{N}_{2}(s)\right) k_{2}(s) \neq 0$. For $\mathbf{v}_{0}=\gamma\left(s_{0}\right)+\mathfrak{u}_{0}\left(\mathbf{N}_{1}\left(s_{0}\right) \pm \mathbf{N}_{2}\left(s_{0}\right)\right)$, we give a function $\mathfrak{G}: \mathbb{R}_{1}^{3} \rightarrow$ $\mathbb{R}$ by $\mathfrak{G}(\mathbf{u})=\left\langle\mathbf{u}-\mathbf{v}_{0}, \mathbf{u}-\mathbf{v}_{0}\right\rangle$, then we have $g_{v_{0}}(s)=\mathfrak{G}(\gamma(s))$. For $\mathbf{v}_{0}=\mathfrak{P} \mathfrak{L} \mathfrak{D}^{ \pm}\left(s_{0}, u_{0}\right)$, since $\mathfrak{L} \mathfrak{C}\left(v_{0}\right)=$ $\mathfrak{G}^{-1}(0)$ and 0 is a regular value of $\mathfrak{G}, g_{v_{0}}(s)$ has the $A_{k}$-singularity at $s_{0}$ if and only if $\gamma$ and $\mathfrak{L} \mathfrak{C}\left(v_{0}\right)$ have $(k+1)$-point contact for $s_{0}$. On the other hand, by Proposition 3.3, the discriminant set $\mathfrak{D}_{G}$ of $G$ is

$$
\mathfrak{D}_{\mathrm{G}}=\left\{\mathbf{v}=\gamma(\mathrm{s})+\mathrm{u}\left(\mathbf{N}_{1}(\mathrm{~s}) \pm \mathbf{N}_{2}(\mathrm{~s})\right) \mid \mathrm{s} \in \mathrm{I}\right\} .
$$

The assertion (A)(1) of Theorem 2.3 follows from Proposition 3.2, Proposition 3.4, and Theorem 3.1. Since the locus of the singularities of CE is locally diffeomorphic to the line, the assertion (A)(2) holds. Since the locus of singularities of $S W$ is $C(2,3,4)$, the assertion (A)(3) holds.

(2) The discriminant set $\mathfrak{D}_{\widetilde{H}}$ of $\widetilde{\mathrm{H}}$ is

$$
\mathfrak{D}_{\widetilde{\mathrm{H}}}=\left\{(\mathbf{v}, \mathrm{u}) \in \mathrm{Q}_{\epsilon}^{2} \times \mathbb{R} \mid \mathbf{u}=\langle\gamma(\mathrm{s}), \mathbf{v}\rangle, \mathbf{v}=\lambda \mathbf{N}_{1}(\mathrm{~s})+\mu \mathbf{N}_{2}(\mathrm{~s}), \delta\left(\mathbf{N}_{1}(\mathrm{~s})\right) \lambda^{2}+\delta\left(\mathbf{N}_{2}(\mathrm{~s})\right) \mu^{2}= \pm 1\right\} .
$$

The assertion (B) of Theorem 2.3 follows from Proposition 3.2, Proposition 3.4, and Theorem 3.1 similar to the assertion (1). 
(3) By Proposition 3.2, the discriminant set $\mathfrak{D}_{\mathrm{H}_{2}}$ of $\mathrm{H}_{2}$ is

$$
\mathfrak{D}_{\mathrm{H}_{2}}=\left\{\mathbf{v}=\mp \mathbf{N}_{1}(\mathrm{~s}) \mid \mathrm{s} \in \mathrm{I}\right\} \text {. }
$$

The assertion (C) of Theorem 2.3 follows from Proposition 3.2, Proposition 3.4, and Theorem 3.1.

(4) By Proposition 3.2, the discriminant set $\mathfrak{D}_{\mathrm{H}_{1}}$ of $\mathrm{H}_{1}$ is

$$
\mathfrak{D}_{\mathrm{H}_{1}}=\left\{\mathbf{v}=\mp \mathbf{N}_{2}(\mathrm{~s}) \mid \mathrm{s} \in \mathrm{I}\right\} \text {. }
$$

The assertion (D) of Theorem 2.3 follows from Proposition 3.2, Proposition 3.4, and Theorem 3.1.

\section{Parallel slant helix}

A regular unit speed spacelike curve $\gamma: \mathrm{I} \rightarrow \mathbb{R}_{1}^{3}$ is called a parallel slant helix according to parallel frame provided that the unit vector $\mathbf{N}_{1}(s)$ of $\gamma$ has constant angle $\theta$ with some fixed unit vector $\mathbf{u}$, that is $\left\langle\mathbf{N}_{1}(\mathrm{~s}), \mathbf{u}\right\rangle=$ constant, this condition is equivalent to $\frac{\mathrm{k}_{2}(\mathrm{~s})}{\mathrm{k}_{1}(\mathrm{~s})}=$ constant. We can get some detail properties of parallel slant helix by the function $\rho(s)$ defined in Section 2, and Corollary 1 in [23].

Proposition 5.1. Let $\gamma: \mathrm{I} \rightarrow \mathbb{R}_{1}^{3}$ be a regular unit speed spacelike curve. We suppose that $\mathrm{k}_{1}(\mathrm{~s}) \neq 0$ and $\rho(\mathrm{s})=0$, then we have the following claims.

(1) $\gamma(\mathrm{s})$ is a parallel slant helix.

(2) The parallel pseudo-spherical Darboux image is a constant map.

(3) The tangent de Sitter indicatrix is a circle in osculating plane.

(4) The parallel rectifying developable of $\boldsymbol{\gamma}$ is a cylindrical surface given by $\boldsymbol{\gamma}(\mathrm{s})+\mathbf{u} \mathbf{e}(\mathrm{s})$, where $\mathbf{e}(\mathrm{s})=\mathbf{d}_{\epsilon}(\mathrm{s})$.

(5) The normal hyperbolic indicatrix is a circle on the pseudo-spheres $\mathrm{H}^{2}(-1)$ and the direction of the circle is given by the constant vector $\mathbf{d}_{\epsilon}(\mathrm{s}) \equiv \mathbf{e}(\mathrm{s})$.

(6) The normal de sitter indicatrix is a circle on the unit spheres $S_{1}^{2}$ and the direction of the circle is also given by the constant vector $\mathbf{d}_{\epsilon}(\mathrm{s}) \equiv \mathbf{e}(\mathrm{s})$.

Proof.

(1) Suppose $\rho(s)=k_{1}^{\prime}(s) k_{2}(s)-k_{1}(s) k_{2}^{\prime}(s)=0$, by straightforward calculations, we have

$$
\left(\frac{k_{2}(s)}{k_{1}(s)}\right)^{\prime}=\frac{k_{2}^{\prime}(s) k_{1}(s)-k_{2}(s) k_{1}^{\prime}(s)}{k_{1}^{2}(s)}=\frac{-\rho(s)}{k_{1}^{2}(s)}=0
$$

This means that $\frac{k_{2}(s)}{k_{1}(s)}=$ constant. By the definition of parallel slant helix, we know that $\gamma(s)$ is a parallel slant helix.

(2) By the claim (2) of Proposition 3.2, we can get claim (2) easily.

(3) By the Corollary 1 in [23], we also can get claim (3).

(4) The claim (4) is clear by definition.

(5) Suppose $\gamma: I \rightarrow \mathbb{R}_{1}^{3}$ is a regular unit speed spacelike curve with timelike $\mathbf{N}_{1}(\mathrm{~s})$ and $k_{1}(\mathrm{~s})>0$. Other cases follow by the similar computation, we omit them here. Since

$$
\mathbf{e}=\frac{-1}{\sqrt{\left|k_{1}^{2}(s)-k_{2}^{2}(s)\right|}}\left(k_{2}(s) \mathbf{N}_{1}(s)+k_{1}(s) \mathbf{N}_{2}(s)\right),
$$

we get

$$
\left\langle\mathbf{e}, \mathbf{N}_{1}\right\rangle=\left\langle\frac{-1}{\sqrt{\left|k_{1}^{2}(s)-k_{2}^{2}(s)\right|}}\left(k_{2}(s) \mathbf{N}_{1}(s)+k_{1}(s) \mathbf{N}_{2}(s)\right), \mathbf{N}_{1}(s)\right\rangle=\frac{\frac{k_{2}}{k_{1}}}{\sqrt{\left|-1+\left(\frac{k_{2}}{k_{1}}\right)^{2}\right|}}=\text { constant. }
$$


So $\cosh (\alpha(s))=\left\langle\mathbf{e}(s), \mathbf{N}_{1}(s)\right\rangle$ is constant. This means that the first Bishop pseudo-spherical indicatrix is a circle on the hyperbolic space $\mathrm{H}^{2}(-1)$ and the direction of the circle is given by the constant vector $\mathbf{d}_{\epsilon}(s) \equiv \mathbf{e}$.

(6) Under the assumptions of (5), we get

$$
\cos (\beta(s))=\left\langle\mathbf{e}(s), \mathbf{N}_{2}(s)\right\rangle=\frac{-1}{\sqrt{\left|-1+\left(\frac{k_{2}}{k_{1}}\right)^{2}\right|}}
$$

is constant. This means that the second Bishop pseudo-spherical indicatrix is a circle on the de Sitter space $S_{1}^{2}$ and the direction of the circle is also given by the constant vector $\mathbf{d}(\mathrm{s}) \equiv \mathbf{e}$. Other cases follow by the similar computation.

\section{Acknowledgment}

The first author was partially supported by the Doctoral Research Fund of Mudanjiang Normal University No. MNUB201510 and the Project of Science and Technology of Heilongjiang Provincial Education Department of China No. UNPYSCT-2015103. The second author was partially supported by the Project of Science and Technology of Mudanjiang Government No. Z2016s0029. The third author was partially supported by NSF of China No. 11671070.

\section{References}

[1] V. I. Arnol'd, Singularities of Caustics and Wave Fronts, Kluwer Academic Publishers, Dordrecht, (1990). 2

[2] R. L. Bishop, There is more than one way to frame a curve, Amer. Math. Monthly, 82 (1975), 246-251. 1

[3] J. W. Bruce, P. J. Giblin, Curves and Singularities, Cambridge Univ. Press, Cambridge, (1992). 3

[4] B. Bukcu, M. K. Karacan, The Bishop Darboux rotation axis of the spacelike curve in Minkowski 3-space, Ege University, J. Fac. Sci., 3 (2007), 1-5. 1

[5] B. Bukcu, M. K. Karacan, Special Bishop motion and Bishop Darboux rotation axis of the space curve, J. Dyn. Syst. Geom. Theor., 6 (2008), 27-34. 1

[6] B. Bukcu, M. K. Karacan, The slant helices according to Bishop frame, Int. J. Comput. Math. Sci., 3 (2009), 67-70. 1

[7] L. Chen, S. Izumiya, A mandala of Legendrian dualities for pseudo-spheres in semi-Euclidean space, Proc. Japan Acad. Ser. A Math. Sci., 85 (2009), 49-54. 2

[8] N. Clauvelin, W. K. Olson, I. Tobias, Characterization of the geometry and topology of DNA pictured as a discrete collection of atoms, J. Chem. Theory Comput., 8 (2012), 1092-1107. 1

[9] C. Y. Han, Nonexistence of rational rotation-minimizing frames on cubic curves, Comput. Aided Geom. Design, 25 (2008), 298-304.

[10] A. J. Hanson, H. Ma, Parallel transport approach to curve framing, Indiana University, Techreports-TR 425, 11 (1995), 3-7. 1

[11] M. K. Karacan, B. Bukcu, N. Yuksel, On the dual Bishop Darboux rotation axis of the dual space curve, Appl. Sci., 10 (2008), 115-120. 1

[12] T. Körpinar, E. Turhan, On characterization of B-canal surfaces in terms of biharmonic B-slant helices according to Bishop frame in Heisenberg group Heis ${ }^{3}$, J. Math. Anal. Appl., 382 (2011), 57-65. 1

[13] L. Kula, N. Ekmekçi, Y. Yayli, K. Ilarslan, Characterizations of slant helices in Euclidean 3-space, Turkish J. Math., 34 (2010), 261-274. 1

[14] L. Kula, Y. Yayli, On slant helix and its spherical indicatrix, Appl. Math. Comput., 169 (2005), 600-607.

[15] H. Liu, D. Pei, Cusps of Bishop spherical indicatrixes and their visualizations, Math. Prob. Eng., 2013 (2013), 11 pages. 2

[16] H. Liu, D. Pei, Singularities of a space curve according to the relatively parallel adapted frame and its visualization, Math. Prob. Eng., 2013 (2013), 12 pages.

[17] H. Liu, D. Pei, Legendrian dualities between spherical indicatrixes of curves and surfaces according to Bishop frame, J. Nonlinear Sci. Appl., 9 (2016), 2875-2887. 1

[18] M. Özdemir, A. A. Ergin, Parallel frames of non-lightlike curves, Missouri J. Math. Sci., 20 (2008), 127-137. 1, 2, 2

[19] D. Pei, T. Sano, The focal developable and the binormal indicatrix of a nonlightlike curve in Minkowski 3-space, Tokyo J. Math., 23 (2000), 211-225. 2

[20] K. Shoeemake, Animating rotation with quaternion curves, ACM SIGGRAPH computer graphics, 19 (1985), $245-254$. 1 
[21] E. Turhan, T. Körpinar, Spacelike elastic biharmonic curves with timelike $M_{1}$ according to Bishop frame in Minkowski 3-space, Palest. J. Math., 1 (2012), 27-31. 1

[22] D. Ünal, İ. Kisi, M. Tosun, Spinor Bishop equations of curves in Euclidean 3-space, Adv. Appl. Clifford Algebras, 23 (2013), 757-765. 1

[23] S. Y1lmaz, Bishop spherical images of a spacelike curve in Minkowski 3-space, Int. J. Phys. Sci., 5 (2010), 898-905. 1, 2, 5, 5

[24] S. Yılmaz, E. Özyılmaz, M. Turgut, New spherical indicatrix and their characterizations, An. Şt. Ovidius Constanţa, 18 (2010), 337-354. 1

[25] S. Y1lmaz, M. Turgut, A new version of Bishop frame and an application to spherical images, J. Math. Anal. Appl., 371 (2010), 764-776. 1

[26] N. Yüksel, The ruled surfaces according to Bishop frame in Minkowski 3-space, Abstr. Appl. Anal., 2013 (2013), 5 pages. 1 